\title{
Polymorphic SNPs, short tandem repeats and structural variants are responsible for differential gene expression across C57BL/6 and C57BL/10 substrains
}

Milad Mortazavi ${ }^{1}$, Yangsu Ren ${ }^{1}$, Shubham Saini ${ }^{2}$, Danny Antaki ${ }^{1,3}$, Celine St. Pierre ${ }^{4}$, April Williams $^{5}$, Abhishek Sohni ${ }^{6}$, Miles Wilkinson ${ }^{6,7}$, Melissa Gymrek ${ }^{2,7,8}$, Jonathan Sebat ${ }^{\star 1,3,7}$, and Abraham A. Palmer ${ }^{\star 1,7}$

${ }^{1}$ Department of Psychiatry, University of California San Diego, La Jolla, CA

2Department of Computer Science and Engineering, University of California San Diego, La Jolla, CA

${ }^{3}$ Department of Cellular and Molecular Medicine and Pediatrics, University of California San Diego, La Jolla, CA

${ }^{4}$ Department of Genetics, Washington University School of Medicine, St. Louis MO ${ }^{5}$ Salk Institute for Biological Studies, La Jolla, CA

${ }^{6}$ Department of Obstetrics, Gynecology and Reproductive Sciences, University of California San Diego, La Jolla, CA

${ }^{7}$ Institute for Genomic Medicine, University of California San Diego, La Jolla, CA ${ }^{8}$ Department of Medicine, University of California San Diego, La Jolla, CA

\section{${ }^{*}$ Corresponding Authors:}

Abraham A. Palmer (aap@ucsd.edu)

Jonathan Sebat (jsebat@ucsd.edu) 


\section{Summary}

Mouse substrains are an invaluable model for understanding disease. We compared C57BL/6J, which is the most commonly used inbred mouse strain, with several closely related substrains. We performed whole genome sequencing and RNAsequencing analysis on 9 C57BL/6 and 5 C57BL/10 substrains. We identified 352,631 SNPs, 109,096 INDELs, 150,344 short tandem repeats (STRs), 3,425 structural variants (SVs) and 2,826 differentially expressed genes (DEGenes) among these 14 strains. 312,981 SNPs (89\%) distinguished the B6 and B10 lineages. These SNPS were clustered into 28 short segments that are likely due to introgressed haplotypes rather than new mutations. Outside of these introgressed regions, we identified $53 \mathrm{SVs}$, protein-truncating SNPs and frameshifting INDELs that were associated with DEGenes. Our results can be used for both forward and reverse genetic approaches, and illustrate how introgression and mutational processes give rise to differences among substrains.

Keywords: C57BL/6, C57BL/10, Reduced Complexity Cross, substrain, mutational processes, gene expression, introgression, Wdfy1, Lpp, Srp54

\section{Introduction}

Since Clarence C. Little generated the C57BL/6 inbred strain a century ago, the C57BL/6J has become the most commonly used inbred mouse strain. Closely-related C57BL/10 substrains ${ }^{1,2}$, which were separated from C57BL/6 in about 1937, are also commonly used in specific fields such as immunology ${ }^{3}$ and muscular dystrophy ${ }^{4}$. The popularity of C57BL strains has led to the establishment of many substrains (defined as $>20$ generations of separation from the parent colony). Among the C57BL/6 branches, the two predominant lineages are based on C57BL/6J (from The Jackson Laboratory; JAX) and C57BL/6N (from the National Institutes of Health; NIH ${ }^{5,6}$ ). Subsequently, several additional substrains have been derived from the JAX and the NIH branches.

Genetic differences between closely-related laboratory strains have been assumed to be the result of accumulated spontaneous mutations ${ }^{7}$. For those that are selectively neutral, genetic drift dictates that some new mutations will be lost, others will 
maintain an intermediate frequency, and others will become fixed, replacing the ancestral allele $^{8}$. Because of historical bottlenecks and small breeding populations over many generations, fixation of new mutations can be relatively rapid.

Numerous studies have reported phenotypic differences among various C57BL/6and C57BL/10-derived substrains, which are likely attributable to genetic variation. For C57BL/6 substrains, these differences include learning behavior ${ }^{9}$, prepulse inhibition ${ }^{10}$, anxiety and depression ${ }^{11}$, fear conditioning ${ }^{12-14}$, glucose tolerance ${ }^{15}$, alcohol-related behaviors $^{16,17}$, and responses to other various drugs ${ }^{18-21}$. For C57BL/10 substrains, these differences include seizure traits ${ }^{22}$ and responses to drugs ${ }^{23}$. Crosses between two phenotypically divergent strains can be used for quantitative trait mapping. Because crosses among closely related substrains segregate fewer variants than crosses of more divergent strains, identification of causal alleles is greatly simplified ${ }^{21}$. Such crosses have been referred to as a reduced complexity cross $(\mathbf{R C C})^{24}$ and have been further simplified by the recent development of an inexpensive microarray explicitly designed for mapping studies that use RCCs ${ }^{25}$.

Whole Genome Sequencing (WGS) technology provides a deep characterization of Single Nucleotide Polymorphisms (SNPs), small insertions and deletions (INDELs), Short Tandem Repeats (STRs), and Structural Variations (SVs). SNPs that differentiate a few of the C57BL/6 substrains have been previously reported ${ }^{21,26}$. While most SNPs are expected to have no functional consequences, a subset will; for example, SNPs in regulatory and coding regions, which can profoundly alter gene expression and function. STRs have never been systematically studied in C57BL substrains. STRs are highly variable elements that play a pivotal role in multiple genetic diseases, population genetics applications, and forensic casework. STRs exhibit rapid mutation rates of $\sim 10^{-5}$ mutations per locus per generation ${ }^{27}$, orders of magnitude higher than that of point mutations $\left(\sim 10^{-}\right.$ $\left.{ }^{8}\right)^{28}$, and are known to play a key role in more than 30 Mendelian disorders ${ }^{29}$; recent evidence has underscored their profound regulatory role suggesting widespread involvement in complex traits ${ }^{30}$. SVs include deletions, duplications, insertions, inversions, and translocations. SVs are individually less abundant than SNPs and STRs, but collectively account for a similar proportion of overall sequence difference between genomes ${ }^{31}$. In addition, SVs can have greater functional consequences because they can 
result in large changes to protein coding exons or regulatory elements ${ }^{32}$. Large SVs among C57BL/6 (but not C57BL10) substrains were identified using array comparative genomic hybridization ${ }^{7}$, and have also been identified in more diverse panels of inbred strains using WGS ${ }^{33}$. Although some genetic variants that differ between closely related C57BL substraits have been previously reported ${ }^{7,34-36}$, a comprehensive, genomewide map of SNPs, INDELs, STRs, SVs and gene expression differences among C57BL6 and C57BL10 substrains does not exist.

In an effort to create such a resource, we performed whole genome sequencing in a single male individual from 9 C57BL/6 and 5 C57BL/10 substrains ( $30 x$ per substrain) and called SNPs, INDELs, STRs and SVs. In addition, to identify functional consequences of these polymorphisms, we performed RNA-sequencing of the hippocampal transcriptome in 6-11 male mice from each substrain, which allowed us to identify genes that were differentially expressed (Figure 1A). This approach has two advantages: first it provides a large number of molecular phenotypes that may be caused by substrain specific polymorphisms. Second, we assumed that the gene expression differences would often reflect the action of cis regulatory variants, making it possible to narrow the number of potentially causal mutations without requiring the creation of intercrosses.

\section{Results}

Processing WGS data, we identified 352,631 SNPs, 109,096 INDELs, 150,344 STRs and 3,425 SVs in nine C57BL/6 and five C57BL/10 substrains. $5.6 \%$ of SNPs and $17.2 \%$ of INDELs are singletons (only occur in one substrain). $89 \%$ of SNPs and $58 \%$ of INDELs separated the C57BL/6 and C57BL/10 branches. The fraction of variants in each category observed in different number of substrains is plotted in Figure S1. RNAsequencing analysis on 106 hippocampal samples identified 16,400 expressed genes and 2,826 DEGenes (17.2\%) in C57BL/6 and C57BL/10 substrains (FDR<0.05). These data are available in the Supplementary material.

\subsection{Genetic evidence for origin of C57BL/6 and C57BL/10 substrain differences}


Figure 1B shows the relationships among C57BL/6 and C57BL/10 substrains based on historical records ${ }^{37-39}$. Figure 1C shows a dendrogram that was produced using SNPS, STRs and bi-allelic SVs. Comparison of these two figures shows that the records about the relationships among C57BL/6 and C57BL/10 substrains are consistent with our sequencing results.

\subsection{Distribution of genomic variants across the genome}

The distribution of variants across the genome is shown in Figure 1D. Several dense clusters of variants common in all categories (SNPS, INDELs, STRs and SVs) are evident (e.g. on chromosomes 4, 8, 11 and 13 for example). The non-uniformity of these polymorphisms was inconsistent with our expectation that polymorphisms were due to new mutations and genetic drift. To further explore this observation, we examined the distribution of SNPs for each of the 14 different substrains (Figure 1E). This figure demonstrates that these clusters consist of a series of highly divergent haplotypes that differentiated the C57BL/6 and C57BL/10 lineages. In total 312,981 SNPs (89\% of SNP variants detected in this study and $99.6 \%$ of the C57BL/6 vs C57BL/10 SNPs) reside in several C57BL/10-specific clusters that represent just $5 \%$ of the genome (28 segments on 11 chromosomes: $1,2,4,6,8,9,11,13,14,15,18)$ with a SNP density of $\sim 1 / 425$ bp. Across the remaining 95\% of the genome SNPs do not appear to be clustered and have a density of $\sim 1 / 67,000 \mathrm{bp}$ (more than 100-fold less dense). We found that many of the SNPs in these intervals were present in the Mouse Genome Informatics (MGI) database $(\underline{\mathrm{URL}})$, which suggested they were not due to new mutations in the C57BL/10 lineage. We used the MGI database to identify strains that were similar to these 28 segments. No single strain matched all 28 segments. However, Figure S2 shows that for 24 of the 28 segments, at least one strain in the database had greater than 90\% concordance (we only considered strains for which a minimum of 300 SNPs was available in that segment). Based on these data, we hypothesize that one or more inbred or outbred mice were accidentally introduced into either the C57BL/6 or C57BL/10 lineage. Another possibility is that their last common ancestor was not fully inbred, and that these regions were differentially fixed after their separation. Most of the concordant strains have domesticus origin; however, two large segments on chromosomes 4 and 11 showed apparent 
musculus origin. Additionally, 9,218 SNPs (2.6\% of all SNPs) did not match the C57BL/6J reference genome (mm10) for all substrains, even for the sample we obtained from C57BL/6J. This may be because the cryopreserved embryo stock used for the modern C57BL/6J mice at JAX are more than 20 generations separated from the samples used to generate the mouse reference genome, $\mathrm{GRCm} 38^{40}$. The remaining SNP appeared to be uniformly distributed and are likely due to new mutations. The distributions of other variants (INDELs, STRs and SVs) mirrored SNPs and are plotted in Figure S3.
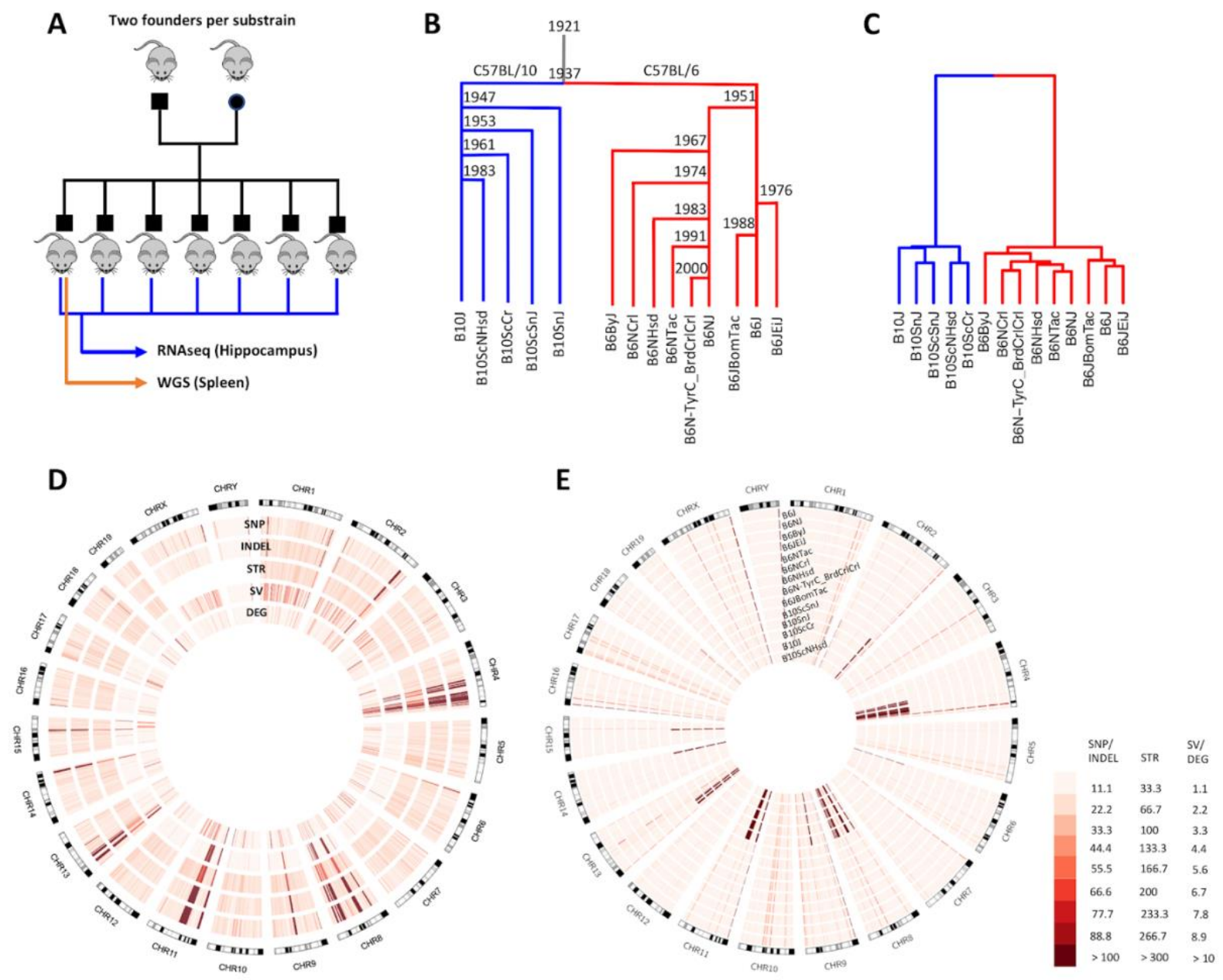

Figure 1. Study design, genetic distance analysis and distribution of genomic variants across the genome. A: This figure shows the design of our study. Mice from nine C57BL/6 and five C57BL/10 substrains were purchased from four vendors. Between six and eleven male offspring from the first generation born in our colony from each substrain were chosen for hippocampal RNA-sequencing. One male offspring per substrain was chosen for whole genome sequencing from spleen tissue. B: Historical development of $\mathrm{C} 57 \mathrm{BL} / 6$ and $\mathrm{C} 57 \mathrm{BL} / 10$ substrains is illustrated as a tree ${ }^{37-39}$. The year in which each substrain is separated from its branch is shown at its junction. C: Dendrogram showing the similarity of the substrains based on genomic variants including: SNPs (LD-pruned, INDELs not included), STRs and SVs. 
D: Circus plot showing the SNPs, INDELs, STRs, SVs, and DEGenes across the genome for 14 C57BL/6 and C57BL/10 substrains. Regions with a high density of polymorphisms (hot spots) on chromosomes 4 , 8, 9, 11 and 13 are obvious. E: Circus plot showing SNPs with non-reference genotypes for each substrain. This plot shows that most hot spots in panel $\mathrm{D}$ are due to regions where all $\mathrm{C} 57 \mathrm{BL} / 6$ differ from all $\mathrm{C} 57 \mathrm{BL} / 10$ substrains. A few regions where all substrains (incuing C57BL/6J) do not match the reference are also evident.

\subsection{Identification of candidate genomic variants causing differential gene expression}

We found that 2,826 of 16,400 genes (17.2\%) were differentially expressed among the 14 substrains (FDR 0.05 ), we call these DEGenes. We assumed that many of the DEGenes were due to local (cis) polymorphisms.

In order to identify genomic variants that might be causally related differential gene expression, we tested all identified variants (SNPs, INDELs, STRs and SVs) in the ciswindow (1 Mb upstream of gene start and $1 \mathrm{Mb}$ downstream of gene end) for association with the corresponding DEGene. Specifically, we tested the association between the cisvariants and the median of DEGene expression by a linear regression test, using Limix ${ }^{41}$. The resulting $p$-values are reported in the Supplementary material.

Our cohort consists of 14 substrains. As expected, all variants with the same strain distribution pattern have identical $p$-values in the association tests. For example, the gene Kcnc2, which had significantly reduced expression in C57BL/6JEiJ (Figure 2A), there was an equally strong correlation with four SNPs, one INDEL and one STR in the cisregion (Figure 2B), and many more variants outside the cis-region (Figure S4). The INDEL was annotated as a frameshift loss-of-function variant by Variant Effect Predictor $(\mathrm{VEP})^{42}$, therefore, it had a strong prior to be the causal variant. Thus, even in this small cohort, we found a number of examples in which a variant within the cis-window had a strong prior and therefore appeared likely to explain a DEGene. We describe several such examples in the next section. However, for the majority of DEGenes, there were no polymorphisms that had strong priors, meaning that any of the variants with the smallest $\mathrm{p}$-values in the cis-window, or a combination of them, or trans-acting variants elsewhere in the genome, could be causal.

2.4 Differential expression of genes is associated with multiple categories of functional variants 
Genomic variants that disrupt protein coding exons or nearby cis-regulatory elements have strong potential to cause differential gene expression. We investigated the causal role of variants in the cis-window by quantifying the strength of effects for multiple functional categories of variants. SNPs and INDELs were annotated using VEP ${ }^{42}$, which identified 555 loss-of-function variants (frameshift, stopgain or splice variant). SVs were annotated by intersecting with the gene features including exons, Transcription Start Site (TSS), Untranslated Regions (UTRs), promoters, enhancers, and introns. When an SV intersected with multiple types of functional elements, it was categorized according to the order mentioned above. The same gene annotations were applied to STRs. Intergenic SVs and STRs, which are defined as those that did not intersect with any gene features, were paired with the gene that had the nearest TSS. In addition, we assessed multiallelic copy number variation of genes by quantifying sequence coverage of all Segmental Duplications mapped to the mm10 reference genome (URL) that intersected with genes.

Genomic variants of the above categories that intersected with DEGenes were tested for association with gene expression by a Linear Mixed Model (LMM) using Limix ${ }^{41}$. We controlled for the complex relationships among inbred strains (a form of population structure) by using a Genomic Relatedness Matrix (GRM), derived from SNP genotypes, as a random effect, and parent strain (C57BL/6 or C57BL/10) as a fixed effect. Figure 2C shows the QQ plot for the $p$-values obtained from the data versus the uniform distribution. The black dots show the deciles of the data in each category. SegDups that intersected genes were strongly correlated with the expression of those genes, as would be expected for gene copy number variation. Loss-of-Function SNPs and INDELs also showed a significant inflation of correlated DEGenes, followed by the genic SVs. The genic STRs showed a slight inflation, which was not as significant as other variant types. The missense SNPS, intergenic SV and intergenic STR p-values followed the uniform distributions.

For each category, the p-values obtained by the LMM model are corrected by the Benjamini-Hochberg procedure to obtain an FDR. We identified 53 significant (FDR < 0.05) associations between DEGens and features, which are reported in Tables S2 and S3. The majority of associations (41 of 53 genes) reflected segmental duplications. In Table S3 we report the genotype pattern in the substrains for each variant; notably, there 
are several clusters of significant associations with the same genotype pattern. For example, one extensive region on Chromosome 2 that clearly distinguishes C57BL/6NJ from all other substrains accounts for 18 of the 53 genes identified. Another cluster with a more complex genotype pattern on Chromosome 4 accounts for 11 of the 53 identified genes.

A

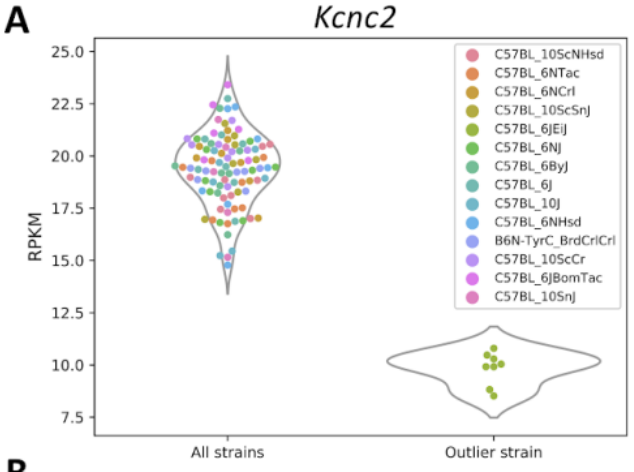

B

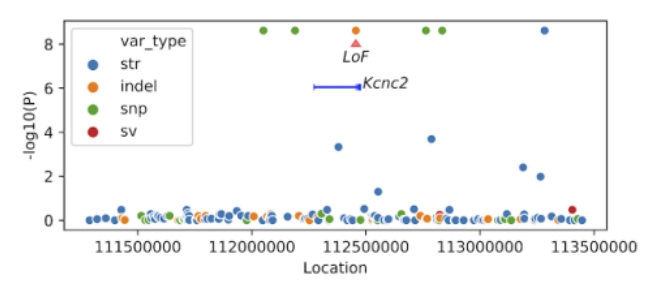

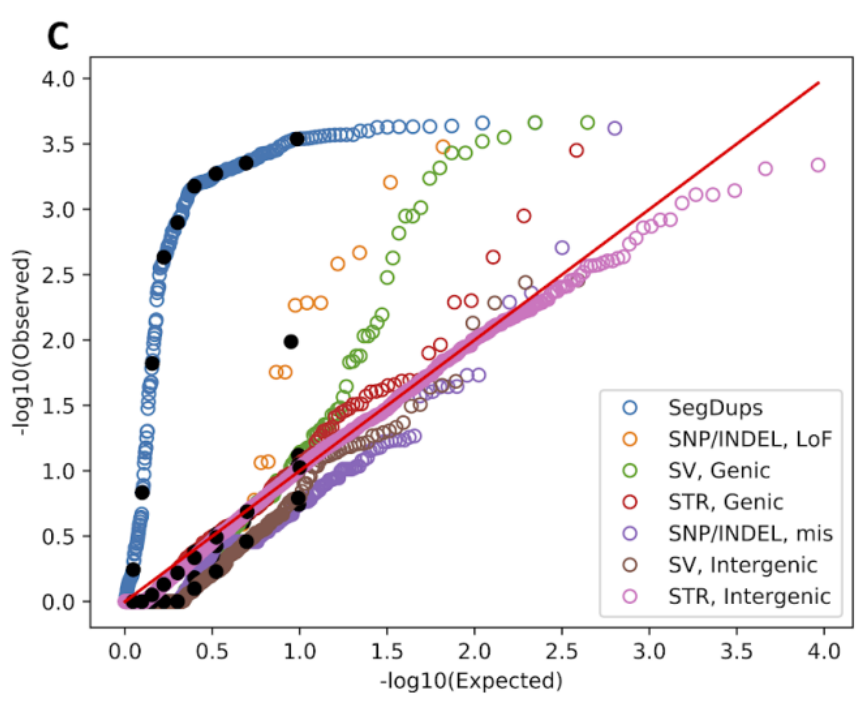

Figure 2. Association of gene expressions with genomic variants. A: Expression of $K c n c 2$ is lower for C57BL/6JEiJ compared to the other substrains. B: Cis-variants of Kcnc2 are tested for association with the median expression by the linear regression model. One INDEL is a frameshift loss-of-function variant and therefore has a strong prior to be the causal variant. In addition to that, four SNPs and one STR also have the same strain distribution pattern, and therefore the same $-\log 10(p)$ value. For most of the DEGenes, no variant belonged to a class that had a strong prior for causality. In those cases, any of the variants with the smallest $p$-values (or a combination thereof) are equally likely to be causal. C: The distribution of the $p$ values of variants in different categories is compared against the uniform distribution in a QQ plot. A Linear Mixed Model is used with the Genomic Relatedness Matrix (GRM) as a random effect to control for population structure and the parental strain (C57BL/6 or C57BL/10) is used as a fixed effect to identify associations within $\mathrm{C} 57 \mathrm{BL} / 6$ and $\mathrm{C} 57 \mathrm{BL} / 10$ substrains. The SegDup category includes associations between the copy number variation of the DEGenes intersecting with SegDup regions (obtained by read depth across the segmental duplication regions of the reference genome) and the gene expression. Lossof-function and missense mutations are two categories of SNP/INDELs. Genic SVs include those intersecting with gene features such as exons, TSS, UTRs, promoters, enhancers and introns, and genic STRs include those intersecting with exons, TSS, 5'UTRs and promoters. Intergenic SVs and STRs are those not intersecting with any gene features, and are paired with a gene with the closest TSS.

\subsection{Distinct mechanisms of differential gene expression caused by SVs}


SVs can affect gene function by (1) varying the dosage of a full-length gene (2) deletion or insertion of exons producing alternative isoforms of a gene, or (3) rearrangement of the cis-regulatory elements of genes. For example, there are three copies of the gene Srp54 in the mouse reference genome, but we found significant variability in the number of copies across the substrains; the number of copies was strongly associated with expression of Srp54 (Figure 3A). Thus, in this example, copy number variation in highly mutable segmental duplication regions is the likely cause of differential gene expression. An example of a SV that likely impacts expression is the $L p p$ gene. The Lpp gene has a tandem duplication of the first two exons in two substrains (C57BL/10ScCr and C57BL/10ScNHsd) that creates two copies of the TSS, which probably accounts for its 2-fold increased expression (Figure 3B).

An intriguing example of altered expression caused by a SV is the Wdfy 1 gene. This gene has a tandem duplication of exons 4-6 in the C57BL/6J substrain, which is also present in the mm10 reference genome. We found this duplication is associated with a paradoxical decrease in Wdfy1 gene expression (Figure 3C). Given that a frameshift is incurred by this tandem duplication, we hypothesized that reduced Wdfy1 expression is caused the nonsense-mediated RNA decay (NMD) pathway. This highly conserved RNAturnover pathway promotes the turnover of mRNAs harboring premature termination codons, including those generated by frameshifts ${ }^{43}$. We reasoned that if a major spliced isoform of Wdfy 1 contains this tandem duplication and associated frameshift, its decay by NMD would be detectable by examining expression in NMD-deficient cells. In support, RNA-seq analysis showed that NMD-deficient (Upf2/--) C57BL/6J ES cells expressed 2fold higher levels of Wdfy1 than control (sibling) C57BL/6J ES cells (Figure 3F). Analysis of splice junctions from RNA-seq analysis confirmed the existence of an aberrant isoform in all C57BL/6J lines that includes splicing of exon 6 to the downstream (duplicated) exon 4 (Figure 3D), which we refer to as the "6a->4b" junction. This splice junction was unique to $\mathrm{C} 57 \mathrm{BL} / 6 \mathrm{~J}$ strains, and the ratio of $6 \mathrm{a}->4 \mathrm{~b}$ to all splice junctions was increased by $\sim 2-$ fold in the Upf2/- ES cells relative to control ES cells (Figure 3E). These results demonstrate that major isoforms transcribed from Wdfy1 in C57BL/6J mice are degraded by NMD, and the transcripts that are retained are alternative splice forms that exclude the $6 a->4 b$ junction. 
bioRxiv preprint doi: https://doi.org/10.1101/2020.03.16.993683; this version posted January 20, 2021. The copyright holder for this preprint (which was not certified by peer review) is the author/funder, who has granted bioRxiv a license to display the preprint in perpetuity. It is made available under aCC-BY-ND 4.0 International license.

A
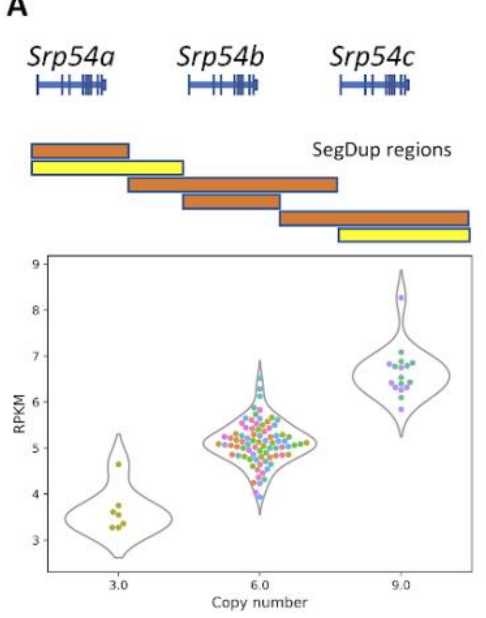

B
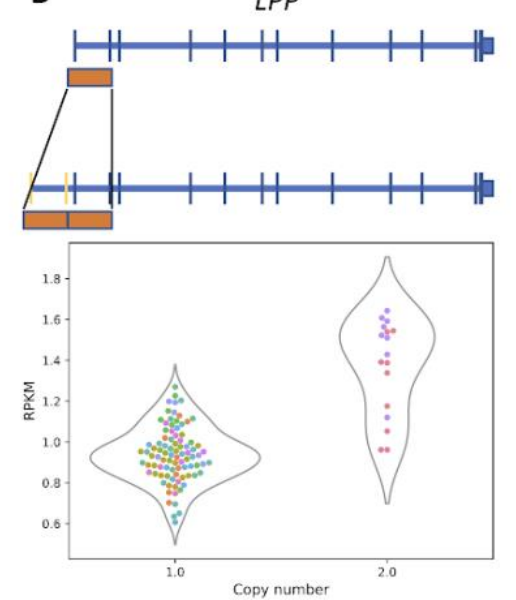

C WDFY1 Reference allele
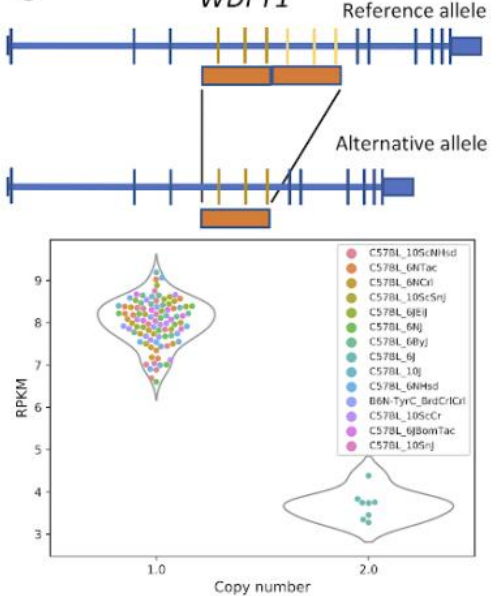

WDFY1

D

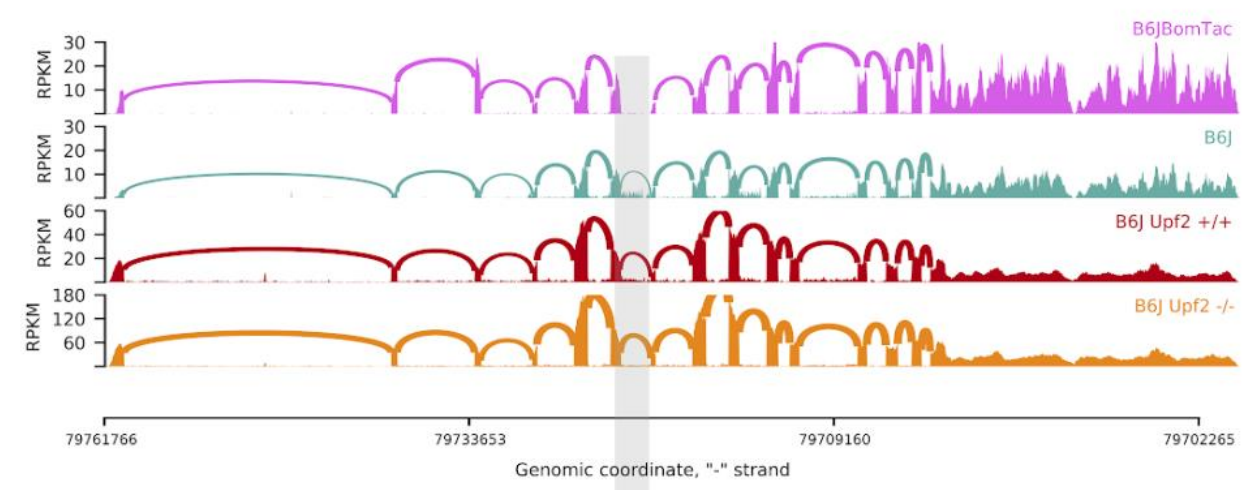

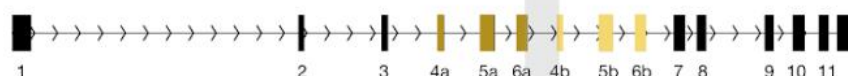

$\mathbf{E}$

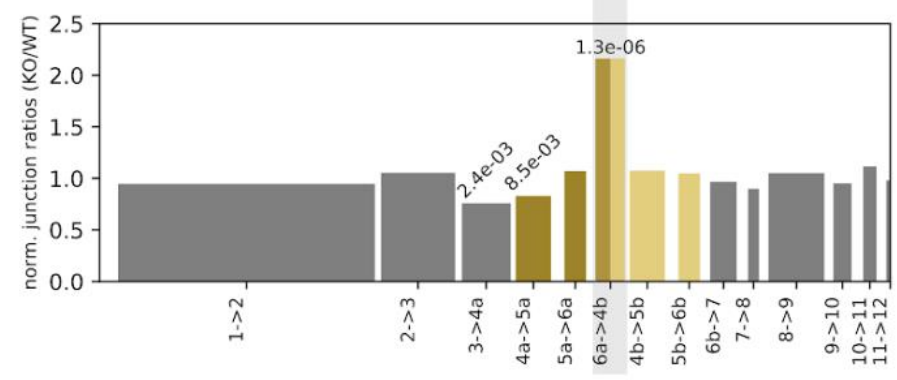

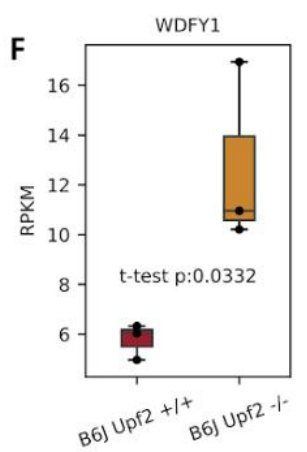

Figure 3. Structural variations affecting gene expression. Structural variations in Srp54, Lpp and Wdfy 1 associate with gene expression of these three genes. A: Various copy numbers in a SegDup region in different substrains is associated with expression of Srp54. The read coverage in these SegDup regions is used to infer the number of copies of the intersecting genes. B: A duplication involving the first two exons of $L p p$ in two substrains of $\mathrm{C} 57 \mathrm{BL} / 10$ is associated with increasing the gene expression. Duplicating the TSS and the promoter site seems to be the cause of enhancing the expression level of Lpp. C: A segmental 
duplication region which intersects with three exons in Wdfy1, and is present in C57BL/6J and the mouse reference genome, is associated with reduction of expression of Wdfy 1 in C57BL/6J. All the other substrains lack this duplication. D: Sashimi plots for C57BL/6BomTac (a closely related substrain to C57BL/6J), C57BL/6J, Upf2+/+, and Upf2/- cell lines from C57BL/6J highlighting the junction between $6 \mathrm{a}$ and $4 \mathrm{~b}$ exons across the segmental duplication region. Since C57BL/6BomTac lacks the duplication, it does not have any junctions between those exons, while the relative number of junctions in the Upf2/- cell line is significantly larger than the other wild type C57BL/6J samples. E: The bar plot shows the ratio of the normalized number of junctions between $6 \mathrm{a}$ and $4 \mathrm{~b}$ exons (normalized by the total number of junctions in each sample) in Upf2 1- over Upf $2^{+/+}$cell lines. It shows a significant increase in the relative number of junctions between the two segmental duplications in the Upf2/- cell line. The numbers on top of the bars show p-values obtained by the Chi-squared test. F: The expression level of Wdfy 1 in the Upf $2^{/-}$cell line is significantly higher than the Upf2 ${ }^{+/+}$cell line. This supports our hypothesis that the reduction of gene expression in C57BL/6J is due to the nonsense mediated decay (NMD) mechanism.

\section{Discussion}

We have performed a large-scale multi-omics analysis of 14 C57BL substrains. We identified 352,631 SNPs, 109,096 INDELs, 150,344 STRs and 3,425 SVs; furthermore, of the 16,400 genes that were expressed in the hippocampus, 2,826 were significantly differentially expressed (FDR<0.05). Unexpectedly, many of the polymorphisms that differentiated the C57BL/6 and C57BL/10 substrains were concentrated in a few haplotypes, comprising just 5\% of the genome. These polymorphisms appear to be due to either introgression of an unrelated individual, or incomplete inbreeding at the time that the C57BL/6 and C57BL/10 lineages diverged. Setting these introgressed regions aside, we tried to identify variants that were causally related to differential gene expression by focusing on the cis regions around DEGenes. This allowed us to identify 53 genes in which a variant with high prior probability to be causal was significantly associated with gene expression. While the majority of these 53 instances were caused by segmental duplications, several of which spanned many adjacent genes, a smaller proportion were due to SVs and INDELs (see Supplemental Tables S2 and S3). Inflation of test statistics for these categories of variants further underscores their likely causal roles and highlights the fact that a relaxed FDR threshold would have identified more than 53 variant/DEGene associations.

An unexpectedly large subset of variants (89\% of all SNPs) were concentrated in 28 highly diverged haplotypes that were present in all C57BL/10 strains and represented just $5 \%$ of the genome. These dense clusters of genetic variation (1 SNP/425 bp) perfectly differentiated C57BL/10 from C57BL/6, and likely reflect introgression from another strain. 
Intriguingly, the smaller haplotypes appeared to be of domesticus origin, and were similar to haplotypes found in multiple non-C57BL inbred strains. The two largest haplotypes appeared to be of musculus origin and were also similar to multiple non-C57BL inbred strains. The exact sequence of events that led to this situation are impossible to deduce, but these patterns are clearly due to breeding errors rather than spontaneous mutations; this conclusion is based on several observations: 1) the density of the polymorphisms, 2) the abrupt boundaries of the regions/haplotypes and 3) the fact that the SNPs in these introgressed regions are found in other inbred strains, which would not be the case if they were due to spontaneous mutations. A previous microarray study performed on 198 inbred mouse strains also identified SNP differences between C57BL/6J and three C57BL/10 substrain (C57BL/10J, C57BL/10ScNJ and C57BL/10ScSnJ) for all the 28 introgressed segments that we identified ${ }^{44,45}$; however, that study did not highlight the significance of that finding, and did not have sufficiently dense coverage to define the boundaries of the introgressed regions. While a majority of C57BL/10-specific genetic variants lie within these introgressed regions, they contained only a small fraction ( 13\%) of DEGenes; however, given that the introgressed regions represent only $5 \%$ of the genome, this is still about 2.6-fold greater density of DEGenes that would be expected if they were randomly distributed across the genome.

Outside of these apparently introgressed regions we identified 37,745 SNPs that were distributed throughout the genome in a Poisson fashion with more than 100-fold lower density ( 1 SNP/67,000 bp). These SNPs are apparently due to the accumulation of new mutations and their identification was the original goal of our study. Dendrograms based on these SNPs recapitulated the historically recorded relationships among the substrains (Figure 1B). For the relatively large number of DEGenes $(>2,000)$ that were located outside of the introgressed regions, we considered the association between different categories of nearby (cis) variants, and expression of DEGenes. Variable copy number segmental duplicated regions were shown to be highly enriched for significant associations as were genic SVs and loss of function SNP/INDELs (Figure 2C).

We presented several examples to highlight how different classes of variants underlie DEGenes. For example, variable copy number segmental duplications led to both increased and decreased expression of Srp54 (Fig 3A). In another example, 
duplication of transcription start sites led to increased expression of $L p p$ (Fig $3 \mathbf{B}$ ). In the case of Wdfy1, duplication of several exons led to down-regulation of expression (Fig 3C), which we showed was due to NMD-mediated mRNA decay (Fig 3D). Wdfy1 was previously reported to be differentially expressed between C57BL/6J and C57BL/6NCrl, and was identified as one of the candidate genes for reduced alcohol preference in $\mathrm{C} 57 \mathrm{BL} / 6 \mathrm{NCrl}^{46}$. This gene is also within the QTL named Emo4 (location: Chr1:68,032,186-86,307,305 bp, 两L); mice which are homozygous for C57BL/6J allele are more active in the open field test. Whether Wdfy1 is actually the cause of either association cannot be resolved by our study.

Despite the numerous examples in which likely causal variants were identified, a majority of the causal variants underlying DEGenes remain unknown. Many are likely to be due to variants in regulatory regions that have not been distinguished from other nearby variants with the same strain distribution pattern (and thus identical p-values). Although we focused on the possibility that DEGenes were due to nearby variants (ciseQTLs), the large fraction of differentially expressed genes (17.2\% of all expressed genes) could indicate that many DEGenes are due to trans-eQTLs. Producing crosses between pairs of strains will be necessary to address the relative importance of cis-versus trans-eQTLs in the observed DEGenes; it is possible that such crosses could identify one or more major trans-regulatory hot spots.

Our results create a resource for future efforts to identify genes and causal polymorphisms that give rise to phenotypic differences among C57BL strains using the increasingly popular reduced complexity cross (RCC) approach in which two phenotypically divergent nearly isogenic inbred substrains are crossed to produce an $\mathrm{F}_{2}$ population $^{24}$. Because of the low density of polymorphisms, identifying the causal allele is much more tractable. For example, the gene Cyfp2 was identified as the cause of differential sensitivity to cocaine and methamphetamine in a cross between C57BL/6J and $\mathrm{C} 57 \mathrm{BL} / 6 \mathrm{~N}^{21}$. In the Supplementary material we have provided genomic variants (SNPs, INDELs, STRs and SVs), differentially expressed genes in the hippocampus, as well as association tests between DEGenes and nearby variants. In addition, we have provided the VEP annotated SNP/INDELs, which distinguishes loss of function, missense and synonymous mutations. Our data also identify some regions that have a high density 
of polymorphisms that may complicate the RCC approach. For example, phenotypic differences between C57BL/6 and C57BL/10 strains might frequently map to the introgressed regions, which have a high density of polymorphisms that would significantly hinder gene identification and negate the advantage of RCCs. Furthermore, crosses between two C57BL/6 or between two C57BL/10 strains may map to large segmental duplication regions such as those on Chromosomes 2 and 4 (see Figure 1E and Supplemental Table S3), which would again hinder gene identification. Thus, one key observation from this study is that genetic differences among C57BL/6 and C57BL/10 strains are not uniformly distributed. Furthermore, our study used a single individual to represent each strain for whole genome sequencing. Therefore, we did not explore the extent to which the polymorphic regions we identified may be segregating versus fixed within each inbred strain. If some of these polymorphic regions are not fixed, it would further complicate the analysis of RCCs.

Whereas the RCCs represent a forward genetic approach (starting with a phenotypic difference, searching for the genetic cause), another novel application of our dataset would be to select two strains that are divergent for a coding or expression difference and to use that cross to study gene function. This reverse genetic approach (starting with a genetic difference, searching for the phenotypic consequences) has not been attempted using closely related substrains, but is conceptually similar to characterization of a knockout mouse. This approach is limited by the available polymorphisms. Although it would be necessary to account for the impact of linked polymorphisms, most of the polymorphisms would be unlinked and would not confound the interpretation of results.

In summary, we have created a dataset that elucidates the differences among C57BL strains and can be used for both forward genetic (RCC) and reverse genetic approaches. We identify previously unknown introgressed segments that differentiate the C57BL/6 and C57BL/10 lineages. Our results can also be used to explore mutational processes and highlight the tendency of inbred strains to change over time due to mutational processes. 
Acknowledgements: M.M., Y.R., C.L.S.P., A.W. and A.A.P. were supported by P50DA037844. Additionally, Y.R. was supported by T32MH018399 and A.W. was supported by T32MH020065.

\section{Author Contributions}

A.P. designed the study. C.L.S.P. performed the animal breeding, dissection and the preparation of WGS and RNASeq libraries. Y.R. and A.W. performed initial analyses of the WGS and RNASeq data. M.M. carried out all statistical genetic and functional genomic analyses. M.G. and S.S. performed STR calling. M.M. and J.S. performed SV calling and analysis of SV eQTLs. M.W. developed the Upf2 mouse model, A.S. derived the ES cells and performed the corresponding RNA-Seq. M.M., J.S., A.P. wrote the paper.

\section{Declaration of interest}

The authors declare no competing interests.

\section{Methods}

\subsection{Mice}

We obtained a panel of $14 \mathrm{C} 57 \mathrm{BL}$ substrains from four vendors. The panel included 9 C57BL/6 substrains: C57BL/6J, C57BL/6NJ, C57BL/6ByJ, C57BL/6NTac, C57BL/6JBomTac, B6N-TyrC/BrdCrCrl, C57BL/6NCrl, C57BL/6NHsd, C57BL/6JEiJ, and 5 C57BL/10 substrans: C57BL/10J, C57BL/10ScCr, C57BL/10ScSnJ, C57BL/10SnJ, C57BL/10ScNHsd (Table 1). All of the substrains were bred for one generation at the University of Chicago before tissue was collected for whole genome sequencing and RNA-sequencing; this avoided gene expression differences that were secondary to environmental differences among the four vendors. All procedures were approved by the University of Chicago IACUC. One hundred and ten male mice in total, with six to eleven mice per substrain, were chosen for RNA-sequencing from hippocampus, and one male mouse per substrain was chosen for whole genome sequencing from spleen (Figure 1A). 
DNA from one male animal per substrain $(n=14)$ was extracted from spleens using a standard "salting-out" protocol. Sequencing libraries were prepared using a TruSeq DNA LT kit, as per the manufacturer's instructions. Subsequently, sequencing data was generated by Novogene at an average depth of $\sim 30 \mathrm{X}$ coverage on an Illumina HiSeq X Ten (paired-end 150bp) (Table 1).

Table 1. Strain IDs, vendors and results from sequencing and RNA-sequencing.

\begin{tabular}{|c|c|c|c|c|c|c|}
\cline { 5 - 7 } \multicolumn{2}{c|}{} & \multicolumn{2}{c|}{ Whole Genome Sequencing } & \multicolumn{2}{c|}{ RNA-sequencing } \\
\hline Strain & Vendor & Strain ID & Reads & Coverage & Average reads & \# samples \\
\hline C57BL/6NTac & Taconic & B6 & $336,470,599$ & 33.65 & $13,459,345$ & 8 \\
\hline C57BL/6NJ & JAX & $\# 005304$ & $333,094,625$ & 33.31 & $15,767,477$ & 9 \\
\hline C57BL/6NHsd & Harlan & $\# 044$ & $349,305,241$ & 34.93 & $14,656,480$ & 7 \\
\hline C57BL/6NCrl & Charles River & $\# 027$ & $301,990,053$ & 30.20 & $19,408,390$ & 8 \\
\hline C57BL/6JEiJ & JAX & $\# 000924$ & $442,604,628$ & 44.26 & $16,334,723$ & 8 \\
\hline C57BL/6JBomTac & Taconic & B6JBom & $309,053,911$ & 30.91 & $17,340,684$ & 7 \\
\hline C57BL/6J & JAX & $\# 000664$ & $326,208,826$ & 32.62 & $18,555,108$ & 8 \\
\hline C57BL/6ByJ & JAX & $\# 001139$ & $294,061,881$ & 29.41 & $15,552,591$ & 9 \\
\hline B6N-TyrC/BrdCrCrl & Charles River & $\# 493$ & $316,655,519$ & 31.67 & $15,139,469$ & 7 \\
\hline C57BL/10SnJ & JAX & $\# 000666$ & $284,191,454$ & 28.42 & $14,012,862$ & 6 \\
\hline C57BL/10ScSnJ & JAX & $\# 000476$ & $285,201,231$ & 28.52 & $18,947,235$ & 7 \\
\hline C57BL/10ScNHsd & Taconic & $\# 046$ & $326,586,425$ & 32.66 & $12,453,407$ & 11 \\
\hline C57BL/10ScCr & JAX & $\# 003752$ & $289,068,061$ & 28.91 & $16,384,792$ & 8 \\
\hline C57BL/10J & JAX & $\# 000665$ & $311,292,991$ & 31.13 & $17,550,456$ & 7 \\
\hline
\end{tabular}

\subsection{RNA-sequencing and data processing}

Total RNA was extracted from 110 hippocampal samples using Trizol reagent (Invitrogen, Carlsbad, CA). RNA was treated with DNase (Invitrogen) and purified using RNeasy columns (Qiagen, Hilden, Germany). RNA-sequencing library prep and sequencing was performed by the University of California San Diego Sequencing Core using Illumina TruSeq prep and Illumina HiSeq 4000 machine (single-end 50bp; Table 1). Reads were mapped to mouse reference transcriptome (mm10) using the spliceaware alignment software HiSat $2^{47}$, and counts were normalized using $\mathrm{HTSeq}^{48}$. Only genes that had at least one Count Per Million (CPM), for at least two samples were included in our analysis. We further removed four outlier samples identified by PCA analysis. This left us with gene expression data for 16,400 genes across 106 samples in 14 substrains.

To identify Differentially Expressed Genes (DEGenes) we performed analysis of variance using the anova function in $R$, and adjusted the $p$-values by computing the false- 
discovery rate (FDR) using the p.adjust function in $\mathrm{R}$, with the Benjamini-Hochberg procedure. We obtained 2,826 DEGenes among C57BL/6 and C57BL/10 substrains combined, 1,210 DEGenes within C57BL/6, and 104 DEGenes within C57BL/10 substrains with FDR $<0.05$.

\subsubsection{Nonsense mediated decay assay}

To determine whether SVs of the Wdfy1 gene in C57BL/6J create novel mRNA isoforms that are degraded by the Nonsense-Mediated Decay (NMD) pathway, we performed RNA-seq on mouse embryonic stem cells (mESCs) from a Upf2/- strain of C57BL/6J that has impaired NMD and control mouse mESCs from C57BL/6J. Samples with an RNA integrity index of >8 (as determined by a BioAnalyzer) were used for RNAseq analysis. The University of California San Diego Sequencing Core performed library preparation using ribosomal RNA depletion protocol followed by paired-end sequencing (100 cycles) using a HiSeq4000. Reads from three replicates of Upf2-- samples and three controls were mapped to the mouse reference genome (mm10) by HiSat2 ${ }^{47}$, and counts were normalized using $\mathrm{HTSeq}^{48}$. We kept all genes with $\mathrm{CPM}>1$ and normalized the counts with edge $R$ function in $R$, however, we only analyzed Wdfy 1 expression in an effort to detect differences in NMD between Upf2-- and control samples.

\subsection{SNPS and INDELS}

We used SpeedSeq ${ }^{49}$ to process the WGS paired-end reads. SpeedSeq uses BWA-mem (v.0.7.8) to map the reads to the $m m 10$ reference genome, SAMBLAST ${ }^{50}$ to mark duplicates, Sambamba ${ }^{51}$ to sort the BAM files, and FreeBayes ${ }^{52}$ to jointly call SNPs and INDELs. INDELs are defined as insertions or deletions which are relatively short in length. The length range for the detected INDELs in our study is between one and 64 base pairs, which is approximately the lower bound for SV length scales. We restricted our analysis to variants that were fixed within individual substrains by including homozygous SNPs and INDELs only, resulting in a callset consisting of 352,631 SNPs and 109,096 INDELs. These variants are provided in the Supplementary material.

When computing the identity-by-state (IBS) matrix for dendrograms, we LD-pruned the SNP panel with Plink ${ }^{53}$ (--indep-pairwise 5050.5 ) yielding 16,739 SNPs. This pruned 
SNP set was augmented by STRs and all bi-allelic SVs, followed by computing the distance matrix with dist and plotting the dendrograms with hclust in $R$ v3.6.1.

\subsection{Short Tandem Repeat (STR)}

We used HipSTR v0.6 with default parameters ${ }^{54}$ to call STRs from mapped reads using the mm10 reference STR set available from the HipSTR website (URL). The reference STR set was generated using Tandem Repeats Finder ${ }^{55}$ allowing a maximum repeat unit length of $6 \mathrm{bp}$. STRs for the substrains were jointly genotyped on a single node of a local server in batches of 500 STRs. Resulting VCF files from each batch were merged to create a genome-wide callset in VCF format. We filtered out calls with missing genotypes, as well as calls with reference alleles for all substrains, resulting in a total of 150,344 polymorphic STRs. The STR calls are available in the Supplementary material.

\subsection{Structural Variations (SV)}

SVs were detected using a combination of approaches. First, we called SVs with LUMPY56 and CNVnator ${ }^{57}$, two complementary methods that rely on discordant and split read signals or coverage respectively. Second, because SV calling accuracy by the above methods is low in regions that are dense in segmental duplications, copy number variation within annotated segmental duplications was quantified directly from coverage, and these coverage values were used for the correlation of gene copy numbers with gene expression.

We filtered out SV calls that overlapped $50 \%$ or more with the gap regions of the mouse reference genome, as well as the calls with length smaller than 50 bp and larger than $1 \mathrm{Mbp}$. A more stringent >1000 bp length filter was applied to CNVnator calls. We then filtered out non-homozygous calls and calls that were homozygous for the alternative allele in all substrains.

Concordant calls from LUMPY and CNVnator with $50 \%$ or greater reciprocal overlap and the same genotypes were merged and the breakpoints reported by LUMPY were used. Consensus calls that overlapped with annotated segmental duplications (SegDup) in the reference genome were excluded, and instead SegDup copy number was assessed directly from read depth signal using mosdepth v0.2.6 ${ }^{58}$ with window size 
$100 \mathrm{bp}$. SegDup annotations from the mm10 genome with at least $98 \%$ similarity were intersected with gene annotations, and the median read coverage across SegDups which intersect with genes was normalized by the median coverage of the corresponding chromosome. These normalized coverage values were used to correlate gene copy numbers with gene expression. The final set of SVs included 3,425 deletions, duplications and inversions in nine C57BL/6 and five C57BL/10 substrains. The distribution of SVs in each category and substrain is summarized in Table S1. The VCF file of the SV calls, and the read coverage data for the SegDup regions are provided in the Supplementary material.

\subsection{Resource availability}

\subsubsection{Lead contact}

Further information and requests for resources should be directed to and will be fulfilled by the Lead Contact, Milad Mortazavi (miladm@alumni.stanford.edu).

\subsubsection{Material availability}

This study does not generate new unique reagents.

\subsubsection{Data and code availability}

The datasets (supplementary material) generated during this study are available at Mendeley Data, DOI: 10.17632/k6tkmm6m5h.1 


\section{Supplemental information}

\begin{tabular}{|c|c|c|c|c|c|c|}
\hline \multirow[b]{2}{*}{ Strain } & \multirow[b]{2}{*}{ Substrain } & \multicolumn{3}{|c|}{ CNVnator } & \multicolumn{2}{|c|}{ Lumpy } \\
\hline & & DEL & DUP & DEL & DUP & INV \\
\hline \multirow{9}{*}{ C57BL/6 } & C57BL/6J & 27 & 461 & 11 & 97 & 2 \\
\hline & C57BL/6NJ & 5 & 456 & 65 & 105 & 2 \\
\hline & C57BL/6ByJ & 11 & 426 & 82 & 90 & 5 \\
\hline & C57BL/6JEiJ & 14 & 469 & 42 & 91 & 4 \\
\hline & C57BL/6NTac & 27 & 465 & 64 & 77 & 4 \\
\hline & C57BL/6NCrl & 9 & 420 & 66 & 92 & 2 \\
\hline & C57BL/6NHsd & 127 & 488 & 73 & 86 & 3 \\
\hline & B6N-TyrC/BrdCrCrl & 7 & 445 & 63 & 96 & 3 \\
\hline & C57BL/6JBomTac & 13 & 447 & 50 & 78 & 3 \\
\hline \multirow{6}{*}{ C57BL/10 } & C57BL/10ScSnJ & 95 & 430 & 1204 & 129 & 15 \\
\hline & C57BL/10SnJ & 89 & 420 & 1195 & 125 & 13 \\
\hline & $\mathrm{C} 57 \mathrm{BL} / 10 \mathrm{ScCr}$ & 269 & 481 & 1196 & 103 & 15 \\
\hline & C57BL/10J & 74 & 437 & 1193 & 115 & 16 \\
\hline & C57BL/10ScNHsd & 72 & 426 & 1192 & 123 & 14 \\
\hline & TOTAL & 448 & 1308 & 1369 & 279 & 21 \\
\hline
\end{tabular}

Table S1. Number of SVs found in substrains of C57BL/6 and C57BL/10. 


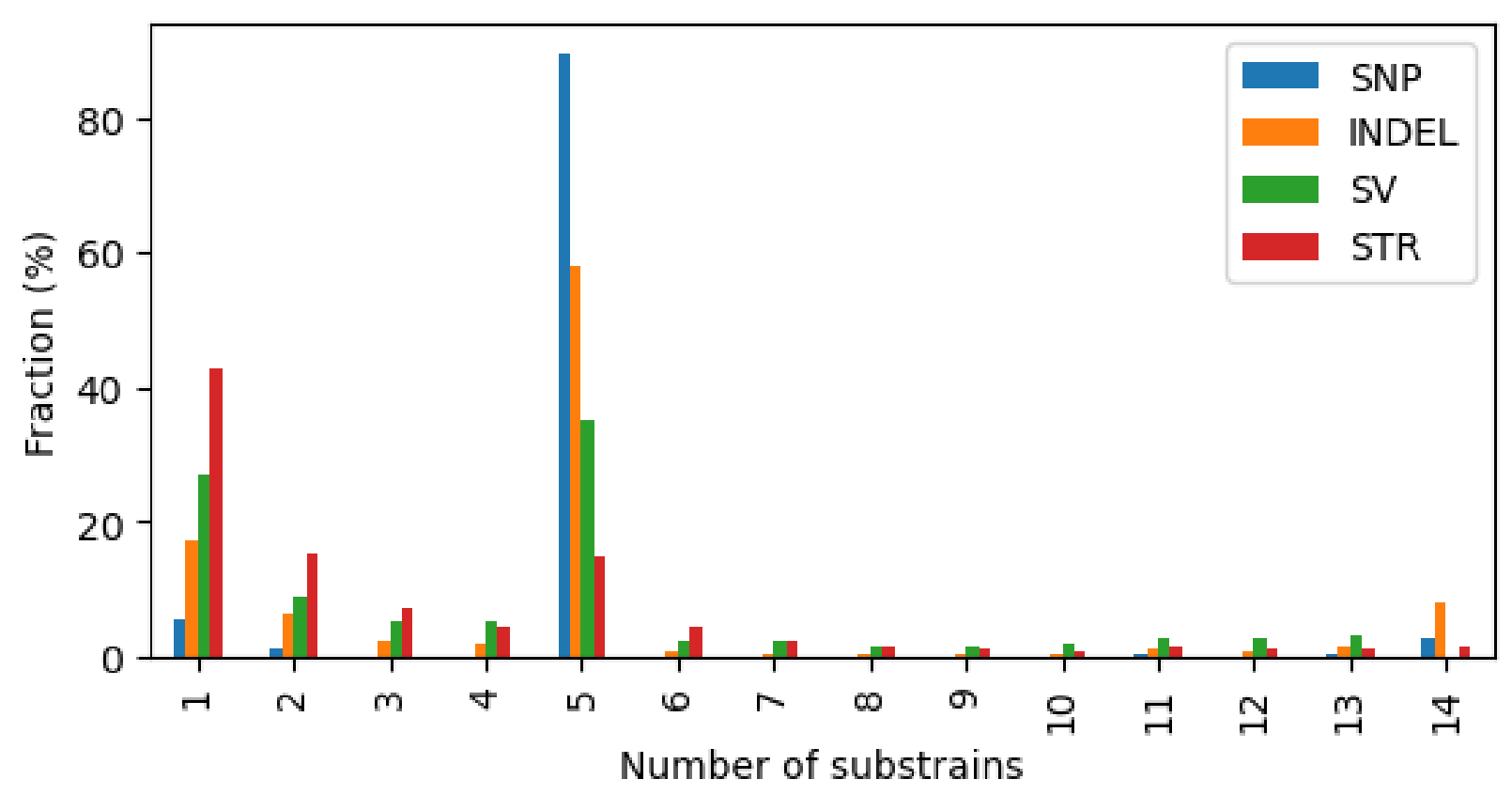

Figure S1. Fraction of variants observed in substrains (five C57BL/10 and nine C57BL/6 substrains) in each variant category. The spike at 5 reflects polymorphisms that separated C57BL/10 $(n=5)$ from C57BL/6 $(n=9)$ substrains. The smaller spike at 14 represents instances where none of the substrains (including $\mathrm{C} 57 \mathrm{BL} / 6 \mathrm{~J}$, which is the basis for $\mathrm{mm} 10$ ) matched the $\mathrm{mm} 10$ reference genome. 


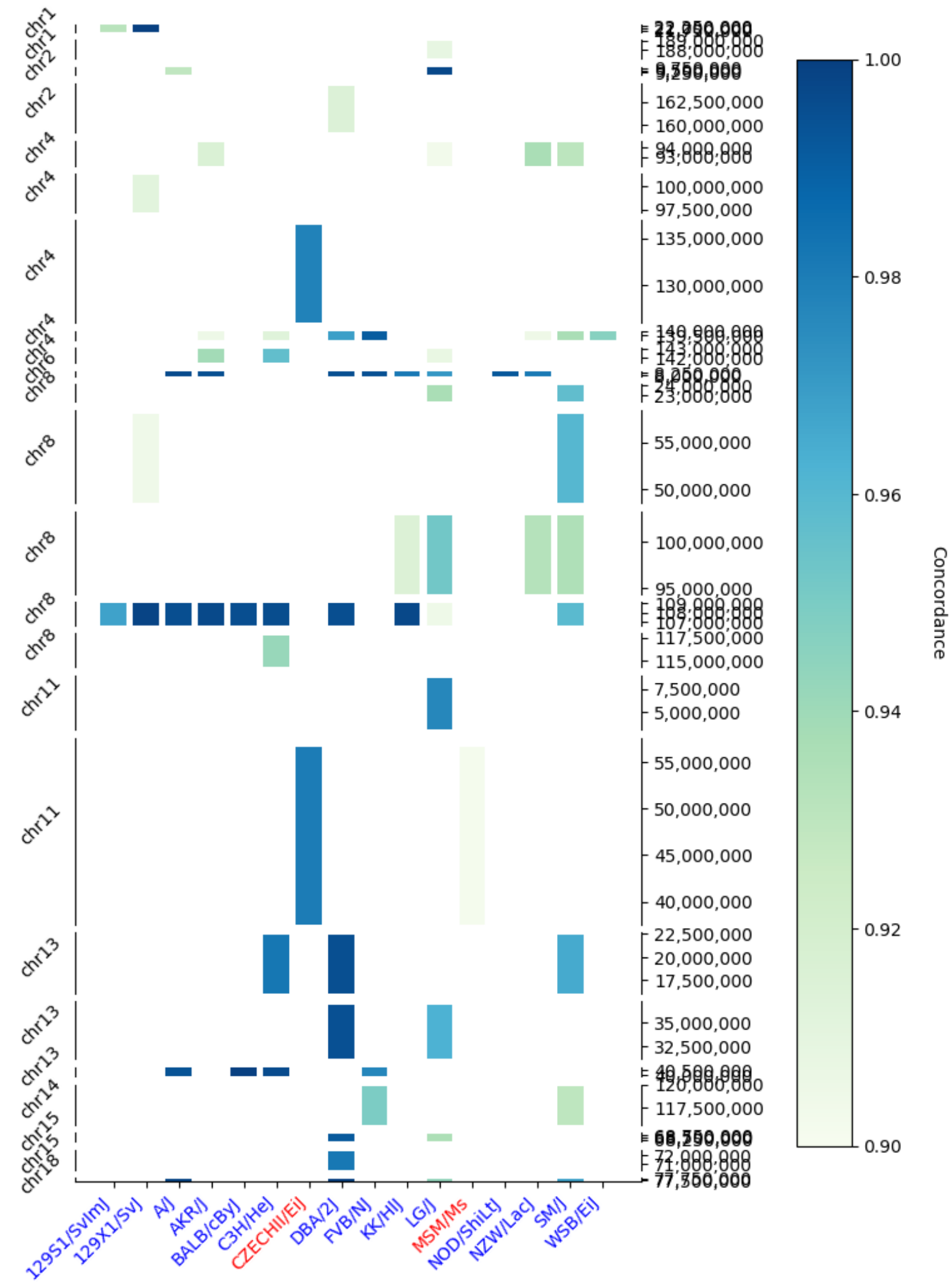

Figure S2. Concordance of 24 C57BL/10-specific haplotypes (SNP hotspots) with SNPs of other strains from domesticus and musculus origin. Y-axis shows segments with C57BL/10-specific SNP hotspots. Xaxis shows strains which have at least 300 common loci and at least $90 \%$ concordance with C57BL/10- 
specific SNPs in each segment. The SNP data for the strains is obtained from MGI (URL). The segments are color coded with the concordance value. The strains on the $\mathrm{x}$-axis are color coded with blue: domesticus origin, and red: musculus origin ${ }^{44}$.
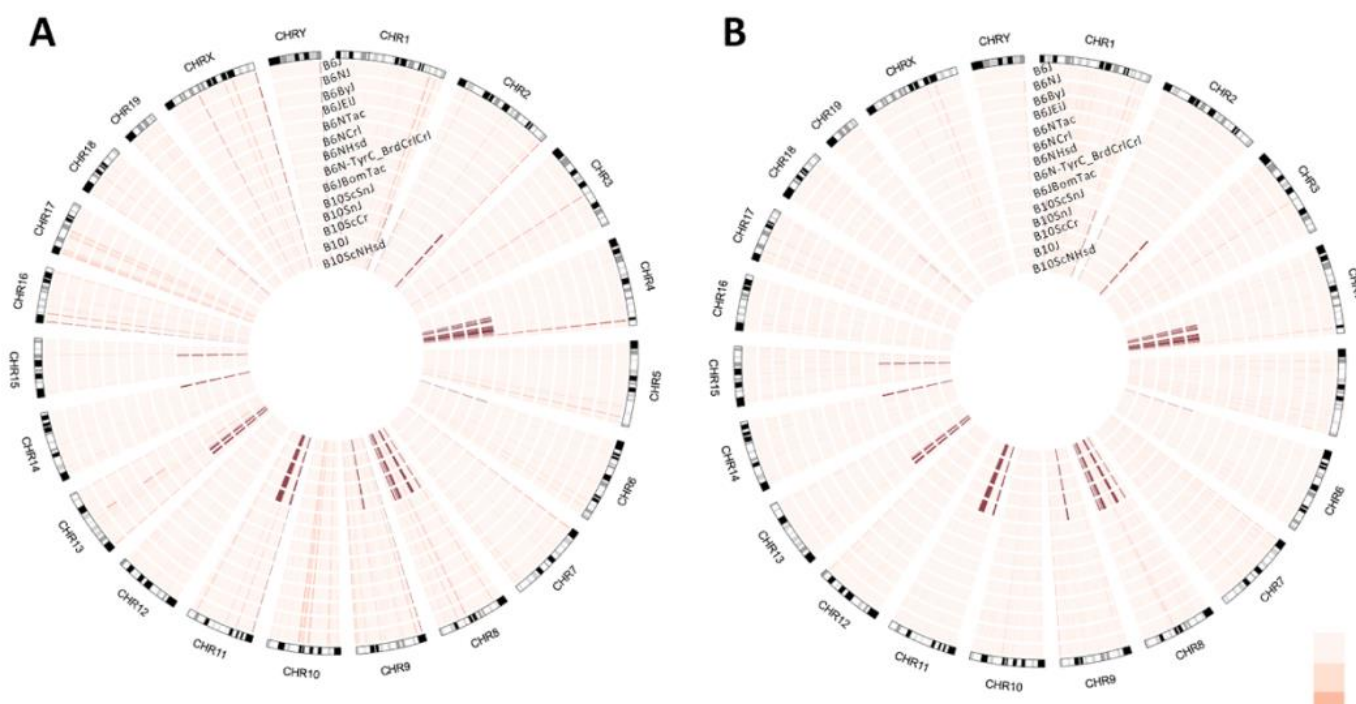

$\begin{array}{llc}\text { SNP/ } & & \text { SV/ } \\ \text { INDEL } & \text { STR } & \text { DEG } \\ & & \\ 11.1 & 33.3 & 1.1 \\ 22.2 & 66.7 & 2.2 \\ 33.3 & 100 & 3.3\end{array}$

C

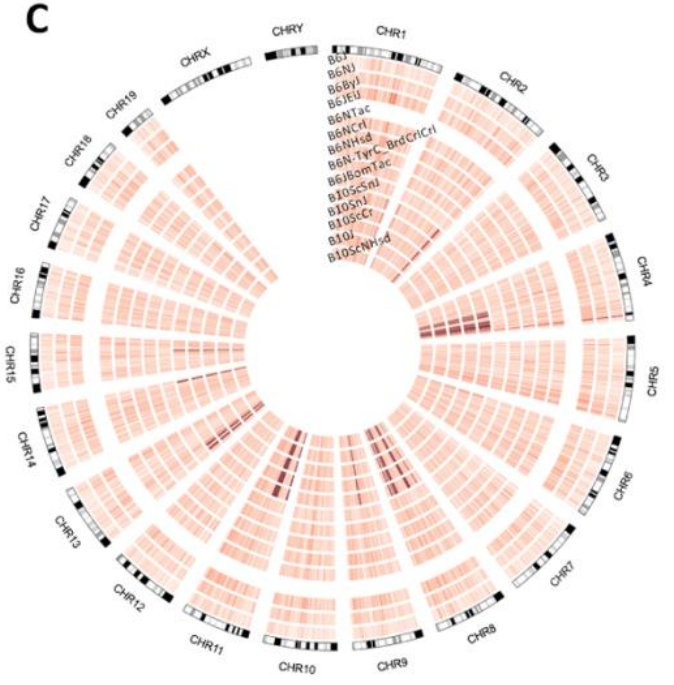

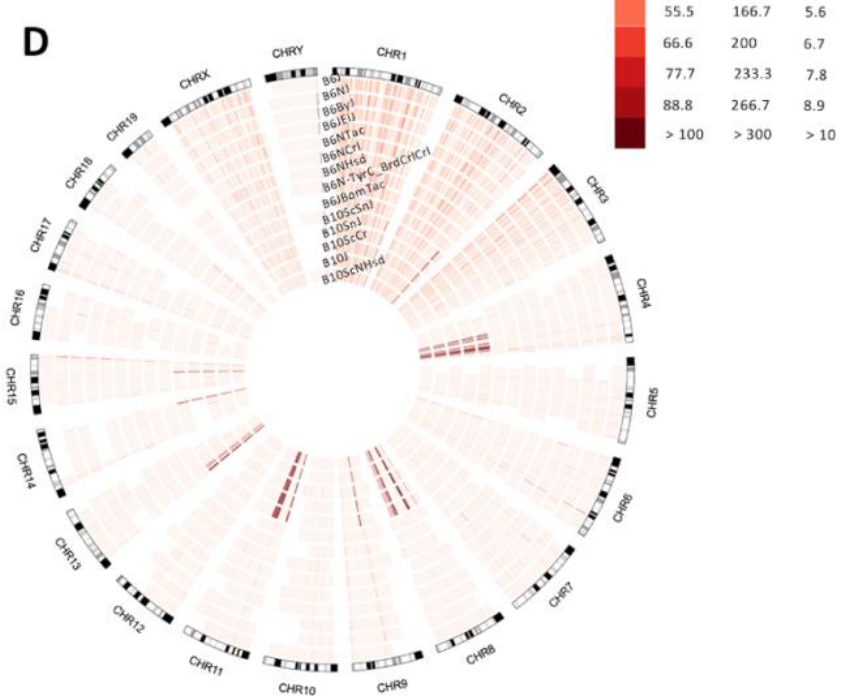

Figure S3. A: SNP distribution, B: INDEL distribution, C: STR distribution, and D: SV distribution for nine C57BL/6 and five C57BL/10 substrains show clusters of variants which are specific to C57BL/10 substrains on chromosomes two, four, eight, nine, eleve, thirteen, fourteen and fifteen. 
A

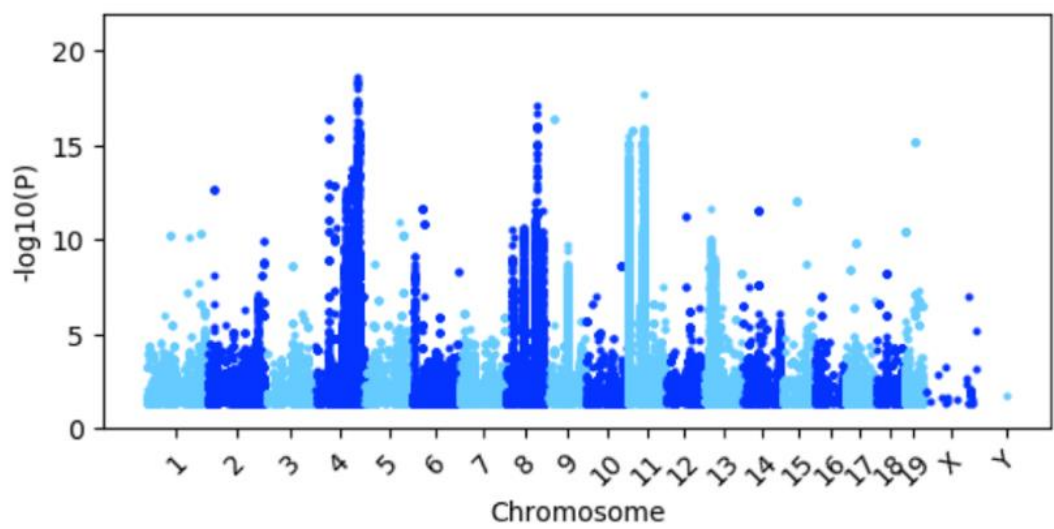

B

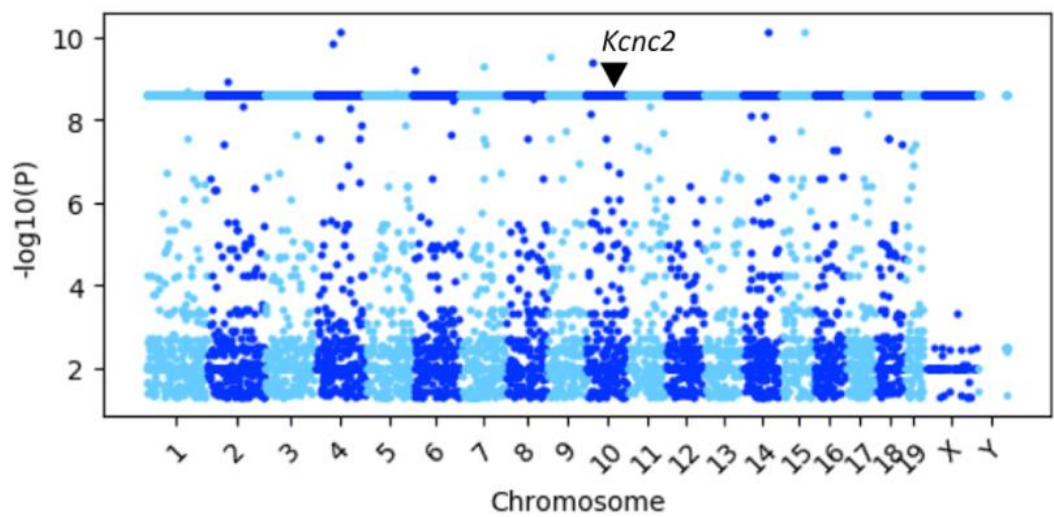

Figure S4. Association tests of median expressions of C57BL/6 and C57BL/10 substrains with all genomic variants (SNPs, INDELs, STRs and SVs) are performed by linear regression model with Limix ${ }^{41}$ A: Association of DEGene median expressions with all variants (SNPs, INDELs, STRs and SVs) in the cisregion defined as $1 \mathrm{Mb}$ upstream and $1 \mathrm{Mb}$ downstream of the DEGene. The $p$-values are plotted at the genomic locations of the corresponding DEGenes. B: Association of Kcnc2 expression with all genomic variants across the genome shows that variants with the same strain distribution pattern have identical $p$ values. The flat horizontal line at about $-\log 10(p)=8.4$ reflects features that have the same strain distribution pattern and therefore all yield identical $p$-values when tested for association with the gene expression data.

\begin{tabular}{|l|c|c|c|c|c|c|}
\hline \multicolumn{1}{|c|}{ Gene } & Variant type & feature & Chrom & Start & End & FDR \\
\hline TIr4 & SV_DEL & exon & chr4 & 66815109 & 66889841 & $2.73 \mathrm{E}-02$ \\
\hline Sh2b3 & SV_DUP & exon & chr5 & 121767693 & 121843545 & $2.73 \mathrm{E}-02$ \\
\hline Arf5 & SV_DUP & exon & chr6 & 28420693 & 28493886 & $3.05 \mathrm{E}-02$ \\
\hline Gcc1 & SV_DUP & exon & chr6 & 28420693 & 28493886 & $3.21 \mathrm{E}-02$ \\
\hline Lpp & SV_DUP & exon & chr16 & 24378934 & 24444153 & $4.53 \mathrm{E}-02$ \\
\hline Btaf1 & SV_DUP & exon & chr19 & 36911370 & 37379559 & $4.53 \mathrm{E}-02$ \\
\hline Fgfbp3 & SV_DUP & exon & chr19 & 36911370 & 37379559 & $2.73 \mathrm{E}-02$ \\
\hline
\end{tabular}




\begin{tabular}{|c|c|c|c|c|c|c|}
\hline Ide & SV_DUP & exon & chr19 & 36911370 & 37379559 & $2.73 \mathrm{E}-02$ \\
\hline Crb1 & INDEL & frameshift & chr1 & 139237085 & 139237087 & $2.02 \mathrm{E}-02$ \\
\hline Tmem108 & INDEL & frameshift & chr9 & 103499764 & 103499767 & $4.25 \mathrm{E}-02$ \\
\hline Kcnc2 & INDEL & frameshift & chr10 & 112455818 & 112455821 & $2.02 \mathrm{E}-02$ \\
\hline Clec16a & INDEL & frameshift & chr16 & 10741835 & 10741842 & 4.25E-02 \\
\hline Wdfy 1 & SD & & chr1 & 79715430 & 79722709 & 1.63E-03 \\
\hline Gm14444 & SD & & chr2 & 174958961 & 175012684 & $1.09 E-02$ \\
\hline Gm14391 & SD & & chr2 & 175176236 & 175476161 & 3.35E-03 \\
\hline Gm4631 & SD & & chr2 & 175176236 & 175476161 & $1.63 \mathrm{E}-03$ \\
\hline Gm6710 & SD & & chr2 & 175600000 & 175900699 & $2.60 \mathrm{E}-03$ \\
\hline Gm14288 & SD & & chr2 & 175675829 & 175804756 & $1.95 \mathrm{E}-03$ \\
\hline Zfp966 & SD & & chr2 & 175926930 & 175959022 & $4.45 \mathrm{E}-03$ \\
\hline Zfp973 & SD & & chr2 & 176598872 & 176698447 & $2.38 \mathrm{E}-03$ \\
\hline Gm14305 & SD & & chr2 & 176702042 & 176745817 & 1.67E-03 \\
\hline Gm14295 & SD & & chr2 & 176742479 & 176876976 & $1.63 \mathrm{E}-03$ \\
\hline Gm14296 & SD & & chr2 & 176834443 & 177060914 & $1.63 E-03$ \\
\hline Gm14401 & SD & & chr2 & 176936335 & 177079562 & $2.50 \mathrm{E}-03$ \\
\hline Gm14410 & SD & & chr2 & 177131439 & 177214443 & $1.63 \mathrm{E}-03$ \\
\hline Gm14418 & SD & & chr2 & 177332425 & 177443112 & $1.63 E-03$ \\
\hline Zfp970 & SD & & chr2 & 177382170 & 177490852 & $1.63 E-03$ \\
\hline Gm14322 & SD & & chr2 & 177714087 & 177825086 & $1.91 \mathrm{E}-03$ \\
\hline Gm14325 & SD & & chr2 & 177837513 & 177900857 & $1.63 \mathrm{E}-03$ \\
\hline Gm14326 & SD & & chr2 & 177900735 & 177965213 & $1.63 \mathrm{E}-03$ \\
\hline Zfp971 & SD & & chr2 & 177954479 & 178027444 & $1.63 E-03$ \\
\hline Gm13302 & SD & & chr4 & 41882892 & 42033952 & 1.63E-03 \\
\hline Gm20878 & SD & & chr4 & 41882892 & 42033952 & 1.63E-03 \\
\hline Gm21093 & SD & & chr4 & 41882892 & 42033952 & 1.63E-03 \\
\hline Gm37530 & SD & & chr4 & 41888084 & 42115916 & 1.63E-03 \\
\hline Gm13306 & SD & & chr4 & 41967677 & 42175681 & 3.35E-03 \\
\hline Gm17167 & SD & & chr4 & 42035272 & 42214541 & 1.63E-03 \\
\hline Gm10600 & SD & & chr4 & 42232133 & 42238731 & 1.63E-03 \\
\hline Gm13301 & SD & & chr4 & 42232133 & 42238731 & 1.63E-03 \\
\hline Gm5859 & SD & & chr4 & 42232133 & 42238731 & 1.63E-03 \\
\hline Gm12394 & SD & & chr4 & 42652191 & 42844101 & $1.63 \mathrm{E}-03$ \\
\hline Gm21955 & SD & & chr4 & 42715617 & 42730325 & 1.34E-02 \\
\hline
\end{tabular}




\begin{tabular}{|l|c|l|c|c|c|c|}
\hline Fam177a & SD & & chr12 & 55078703 & 55124935 & $1.63 \mathrm{E}-03$ \\
\hline Srp54c & SD & & chr12 & 55200000 & 55291223 & $1.63 \mathrm{E}-03$ \\
\hline Gm3636 & SD & & chr14 & 6654813 & 6774339 & $1.63 \mathrm{E}-03$ \\
\hline Gm3667 & SD & & chr14 & 6838781 & 6891591 & $4.45 \mathrm{E}-03$ \\
\hline Gm3739 & SD & & chr14 & 7208412 & 7350351 & $3.87 \mathrm{E}-03$ \\
\hline Tmem181a & SD & & chr17 & 6253548 & 6260484 & $3.14 \mathrm{E}-02$ \\
\hline Tmem181b-ps & SD & & chr17 & 6429915 & 6529109 & $1.63 \mathrm{E}-03$ \\
\hline Dynlt1c & SD & & chr17 & 6497992 & 6645878 & $1.05 \mathrm{E}-02$ \\
\hline Ermard & SD & & chr17 & 15014588 & 15045963 & $4.33 \mathrm{E}-03$ \\
\hline G530011006Rik & SD & & chrX & 169969770 & 170000000 & $2.95 \mathrm{E}-02$ \\
\hline Gm15726 & SD & & chrX & 169969770 & 170000000 & $2.07 \mathrm{E}-02$ \\
\hline
\end{tabular}

Table S2. Significant associations between DEGene expression and large effect variants with FDR $<0.05$. A linear mixed model is used with a Genomic Relatedness Matrix (GRM) to control for population structure as a random effect and parental strain (C57BL/6 versus $\mathrm{C} 57 \mathrm{BL} / 10)$ as a fixed effect to identify associations within C57BL/6 and C57BL/10 substrains. Gene name, variant type, intersection feature, variant chromosome, start, end and FDR are reported for each case. The bold lines separate different variant types, structural variations (SV), SNP/INDEL and copy number variations in segmental duplications (SD).

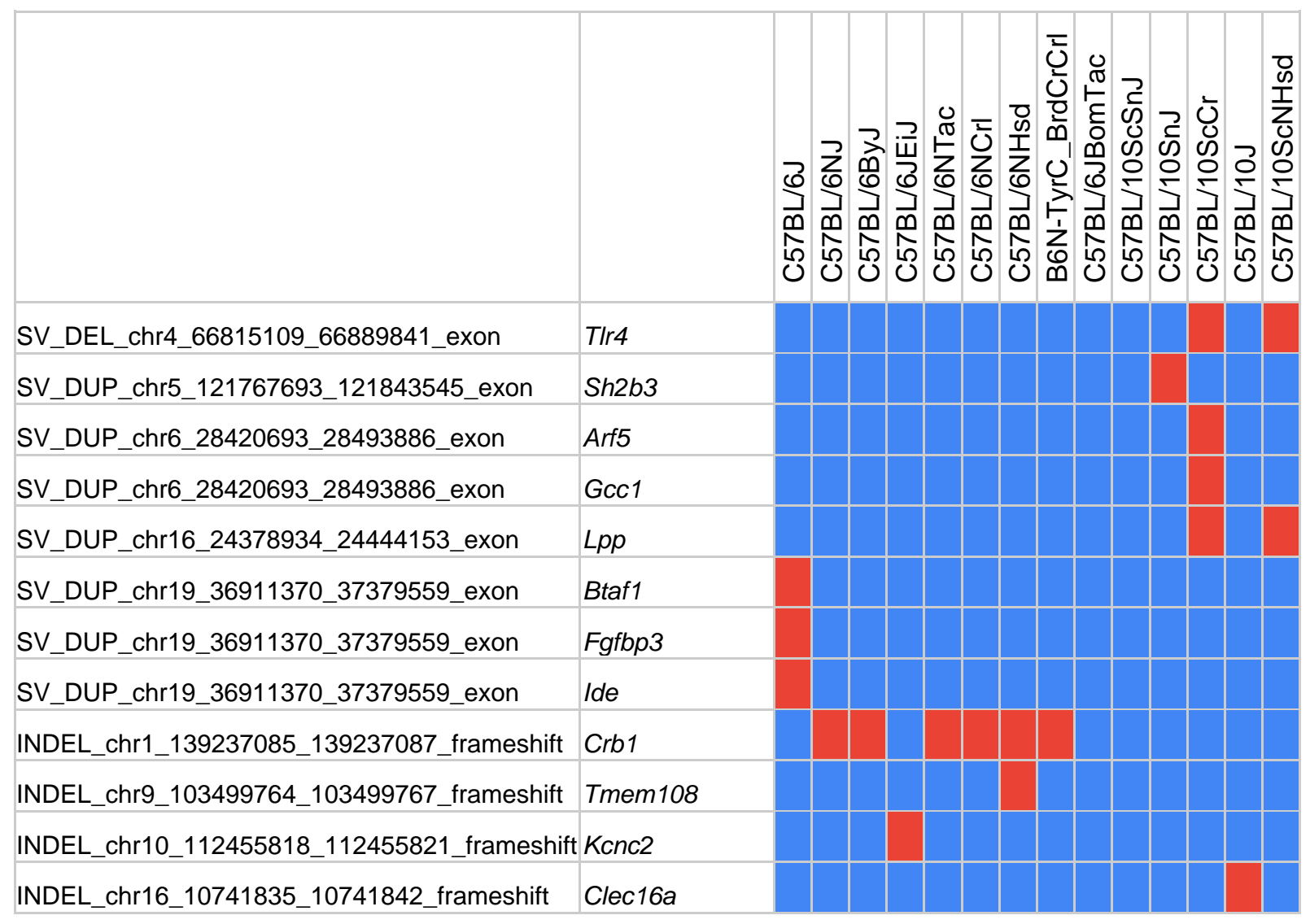




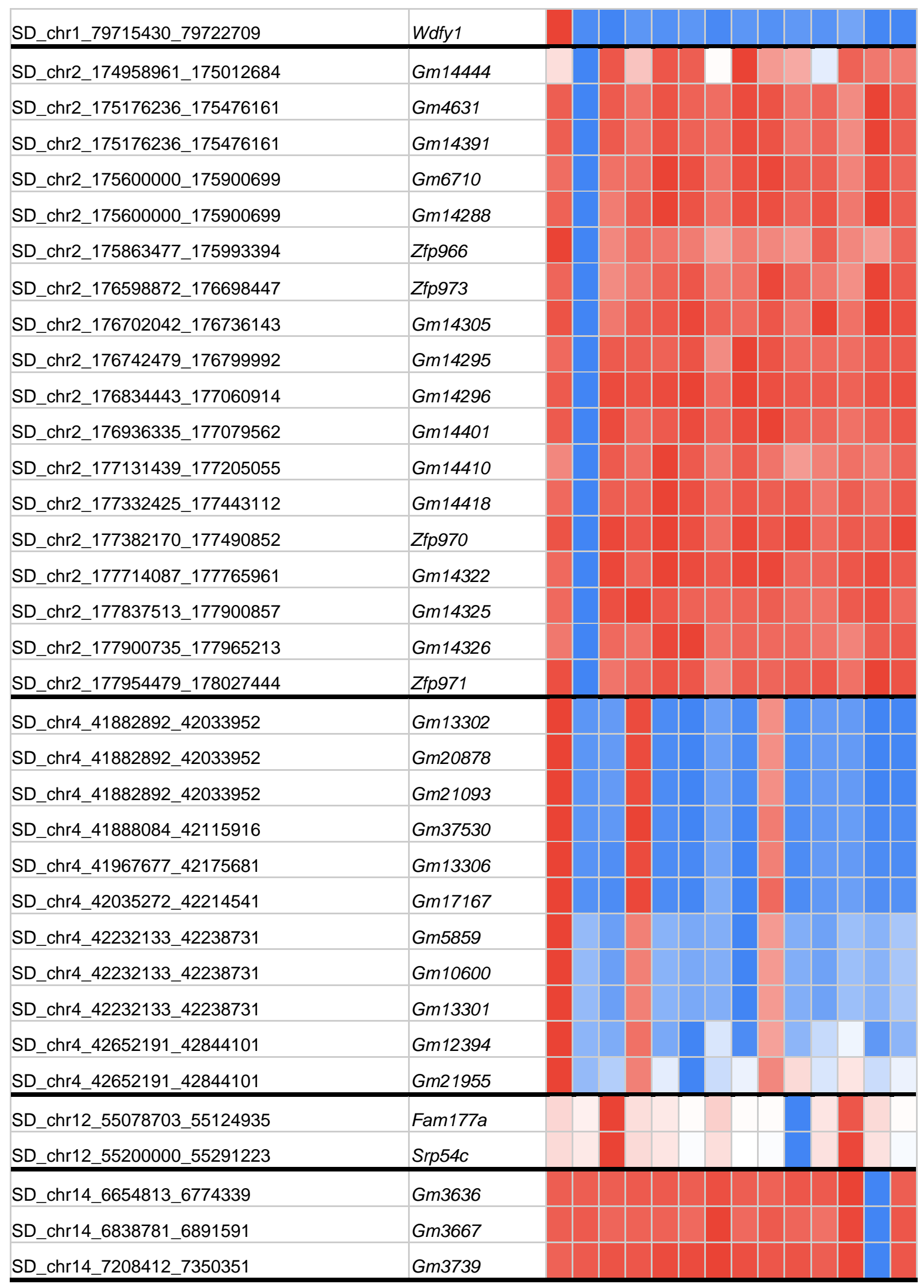


bioRxiv preprint doi: https://doi.org/10.1101/2020 03.16 .993683 ; this version posted January 20,2021 . The copyright holder for this preprint (which was not certified by peer review) is the author/funder, who has granted bioRxiv a license to display the preprint in perpetuity. It is made available under aCC-BY-ND 4.0 International license.

\begin{tabular}{|l|l|l|l|l|}
\hline SD_chr17_6253548_6260484 & Tmem181a & & \\
\hline SD_chr17_6325771_6473130 & Tmem181b-ps & & & \\
\hline SD_chr17_6497992_6645878 & Dynlt1c & & \\
\hline SD_chr17_15014588_15045963 & Ermard & & \\
\hline SD_chrX_169969770_170000000 & G530011006Rik & & \\
\hline SD_chrX_169969770_170000000 & Gm15726 & & \\
\hline
\end{tabular}

Table S3. Genotype patterns for variants with significant association with DEGene expression. For bi-allelic variants (SVs and SNP/INDELs), red and blue colors represent two genotypes, while for multiallelic copy number variants the normalized read depth varies between 0 and 1 where 0: blue, 0.5: white, and 1: red represent three genotypes. The same genotype patterns are clustered together for chromosomes such as 2 and 4, which shows that nearby genes in these regions have been affected by the same copy number variation patterns. Bold horizontal lines segregate nearby variants with similar genotype patterns. 


\section{References}

1. Festing, M.F.W. (1979). Inbred strains in biomedical research (United Kingdom: Macmillan Education, Limited).

2. Lyon, M.F., Searle, A.G., and International Committee on Standardized Genetic Nomenclature for Mice. (1989). Genetic variants and strains of the laboratory mouse, 2nd edn (Oxford England ; New York; Stuttgart: Oxford University Press ; G. Fischer Verlag).

3. von Kockritz-Blickwede, M., Rohde, M., Oehmcke, S., Miller, L.S., Cheung, A.L., Herwald, H., Foster, S., and Medina, E. (2008). Immunological mechanisms underlying the genetic predisposition to severe Staphylococcus aureus infection in the mouse model. The American journal of pathology 173, 1657-1668.

4. Kincaid, A. (2007). Muscular Dystrophy. In xPharm: The Comprehensive Pharmacology Reference, S.J.E.a.D.B. Bylund, ed. (Elsevier Inc.).

5. Bailey, D.W. (1978). Sources of Subline Divergence and Their Relative Importance for Sublines of Six Major Inbred Strains of Mice. In Origins of inbred mice, H.C. Morse, ed. (New York: Academic Press).

6. Altman, P.L., and Katz, D.D. (1979). Inbred and genetically defined strains of laboratory animals (Bethesda, Md.: Federation of American Societies for Experimental Biology).

7. Egan, C.M., Sridhar, S., Wigler, M., and Hall, I.M. (2007). Recurrent DNA copy number variation in the laboratory mouse. Nature genetics 39, 1384-1389.

8. Reed, C., Baba, H., Zhu, Z., Erk, J., Mootz, J.R., Varra, N.M., Williams, R.W., and Phillips, T.J. (2017). A Spontaneous Mutation in Taar1 Impacts Methamphetamine-Related Traits Exclusively in DBA/2 Mice from a Single Vendor. Frontiers in pharmacology 8, 993.

9. Clapcote, S.J., and Roder, J.C. (2004). Survey of embryonic stem cell line source strains in the water maze reveals superior reversal learning of 129S6/SvEvTac mice. Behavioural brain research 152, 35-48.

10. Grottick, A.J., Bagnol, D., Phillips, S., McDonald, J., Behan, D.P., Chalmers, D.T., and Hakak, Y. (2005). Neurotransmission- and cellular stress-related gene expression associated with prepulse inhibition in mice. Brain research Molecular brain research 139, 153-162.

11. Mayorga, A.J., and Lucki, I. (2001). Limitations on the use of the C57BL/6 mouse in the tail suspension test. Psychopharmacology 155, 110-112.

12. Radulovic, J., Kammermeier, J., and Spiess, J. (1998). Generalization of fear responses in C57BL/6N mice subjected to one-trial foreground contextual fear conditioning. Behavioural brain research 95, 179-189.

13. Stiedl, O., Radulovic, J., Lohmann, R., Birkenfeld, K., Palve, M., Kammermeier, J., Sananbenesi, F., and Spiess, J. (1999). Strain and substrain differences in context- and tone-dependent fear conditioning of inbred mice. Behavioural brain research 104, 1-12.

14. Siegmund, A., Langnaese, K., and Wotjak, C.T. (2005). Differences in extinction of conditioned fear in C57BL/6 substrains are unrelated to expression of alpha-synuclein. Behavioural brain research 157, 291298.

15. Toye, A.A., Lippiat, J.D., Proks, P., Shimomura, K., Bentley, L., Hugill, A., Mijat, V., Goldsworthy, M., Moir, L., Haynes, A., et al. (2005). A genetic and physiological study of impaired glucose homeostasis control in C57BL/6J mice. Diabetologia 48, 675-686.

16. Khisti, R.T., Wolstenholme, J., Shelton, K.L., and Miles, M.F. (2006). Characterization of the ethanoldeprivation effect in substrains of C57BL/6 mice. Alcohol 40,119-126.

17. Green, M.L., Singh, A.V., Zhang, Y., Nemeth, K.A., Sulik, K.K., and Knudsen, T.B. (2007). Reprogramming of genetic networks during initiation of the Fetal Alcohol Syndrome. Developmental dynamics : an official publication of the American Association of Anatomists 236, 613-631.

18. Diwan, B.A., and Blackman, K.E. (1980). Differential susceptibility of 3 sublines of C57BL/6 mice to the induction of colorectal tumors by 1,2-dimethylhydrazine. Cancer letters 9, 111-115.

19. Roth, D.M., Swaney, J.S., Dalton, N.D., Gilpin, E.A., and Ross, J., Jr. (2002). Impact of anesthesia on cardiac function during echocardiography in mice. American journal of physiology Heart and circulatory physiology 282, H2134-2140.

20. Kumar, V., Kim, K., Joseph, C., Kourrich, S., Yoo, S.H., Huang, H.C., Vitaterna, M.H., de Villena, F.P., Churchill, G., Bonci, A., et al. (2013). C57BL/6N mutation in cytoplasmic FMRP interacting protein 2 regulates cocaine response. Science 342, 1508-1512. 
21. Akinola, L.S., McKiver, B., Toma, W., Zhu, A.Z.X., Tyndale, R.F., Kumar, V., and Damaj, M.I. (2019). C57BL/6 Substrain Differences in Pharmacological Effects after Acute and Repeated Nicotine Administration. Brain sciences 9.

22. Kadiyala, S.B., Papandrea, D., Herron, B.J., and Ferland, R.J. (2014). Segregation of seizure traits in C57 black mouse substrains using the repeated-flurothyl model. PloS one 9, e90506.

23. Markham, B.E., Kernodle, S., Nemzek, J., Wilkinson, J.E., and Sigler, R. (2015). Chronic Dosing with Membrane Sealant Poloxamer 188 NF Improves Respiratory Dysfunction in Dystrophic Mdx and Mdx/Utrophin-/- Mice. PloS one 10, e0134832.

24. Bryant, C.D., Smith, D.J., Kantak, K.M., Nowak, T.S., Jr., Williams, R.W., Damaj, M.I., Redei, E.E., Chen, H., and Mulligan, M.K. (2020). Facilitating Complex Trait Analysis via Reduced Complexity Crosses. Trends in genetics : TIG 36, 549-562.

25. Sigmon, J.S., Blanchard, M.W., Baric, R.S., Bell, T.A., Brennan, J., Brockmann, G.A., Burks, A.W., Calabrese, J.M., Caron, K.M., Cheney, R.E., et al. (2020). Content and Performance of the MiniMUGA Genotyping Array: A New Tool To Improve Rigor and Reproducibility in Mouse Research. Genetics 216, 905-930.

26. Keane, T.M., Goodstadt, L., Danecek, P., White, M.A., Wong, K., Yalcin, B., Heger, A., Agam, A., Slater, G., Goodson, M., et al. (2011). Mouse genomic variation and its effect on phenotypes and gene regulation. Nature 477, 289-294.

27. Mitra, I., Huang, B., Mousavi, N., Ma, N., Lamkin, M., Yanicky, R., Shleizer-Burko, S., Lohmueller, K.E., and Gymrek, M. (2020). Genome-wide patterns of de novo tandem repeat mutations and their contribution to autism spectrum disorders. bioRxiv 2020.03.04.974170 https://doi.org/10.1101/2020.03.04.974170.

28. Kong, A., Frigge, M.L., Masson, G., Besenbacher, S., Sulem, P., Magnusson, G., Gudjonsson, S.A., Sigurdsson, A., Jonasdottir, A., Jonasdottir, A., et al. (2012). Rate of de novo mutations and the importance of father's age to disease risk. Nature $488,471-475$.

29. Hannan, A.J. (2018). Tandem repeats mediating genetic plasticity in health and disease. Nature reviews Genetics 19, 286-298.

30. Fotsing, S.F., Margoliash, J., Wang, C., Saini, S., Yanicky, R., Shleizer-Burko, S., Goren, A., and Gymrek, M. (2019). The impact of short tandem repeat variation on gene expression. Nature genetics 51, $1652-$ 1659.

31. Chaisson, M.J.P., Sanders, A.D., Zhao, X., Malhotra, A., Porubsky, D., Rausch, T., Gardner, E.J., Rodriguez, O.L., Guo, L., Collins, R.L., et al. (2019). Multi-platform discovery of haplotype-resolved structural variation in human genomes. Nature communications 10, 1784.

32. Hurles, M.E., Dermitzakis, E.T., and Tyler-Smith, C. (2008). The functional impact of structural variation in humans. Trends in genetics : TIG 24, 238-245.

33. Yalcin, B., Wong, K., Agam, A., Goodson, M., Keane, T.M., Gan, X., Nellaker, C., Goodstadt, L., Nicod, J., Bhomra, A., et al. (2011). Sequence-based characterization of structural variation in the mouse genome. Nature 477, 326-329.

34. Quinlan, A.R., Clark, R.A., Sokolova, S., Leibowitz, M.L., Zhang, Y., Hurles, M.E., Mell, J.C., and Hall, I.M. (2010). Genome-wide mapping and assembly of structural variant breakpoints in the mouse genome. Genome research 20, 623-635.

35. Simon, M.M., Greenaway, S., White, J.K., Fuchs, H., Gailus-Durner, V., Wells, S., Sorg, T., Wong, K., Bedu, E., Cartwright, E.J., et al. (2013). A comparative phenotypic and genomic analysis of C57BL/6J and C57BL/6N mouse strains. Genome biology 14, R82.

36. Doran, A.G., Wong, K., Flint, J., Adams, D.J., Hunter, K.W., and Keane, T.M. (2016). Deep genome sequencing and variation analysis of 13 inbred mouse strains defines candidate phenotypic alleles, private variation and homozygous truncating mutations. Genome biology 17, 167.

37. Beck, J.A., Lloyd, S., Hafezparast, M., Lennon-Pierce, M., Eppig, J.T., Festing, M.F., and Fisher, E.M. (2000). Genealogies of mouse inbred strains. Nature genetics 24, 23-25.

38. Charles River Laboratories. Available from: https:/www.criver.com/

39. Jackson Laboratory. Available from: https://www.jax.org/

40. Sarsani, V.K., Raghupathy, N., Fiddes, I.T., Armstrong, J., Thibaud-Nissen, F., Zinder, O., Bolisetty, M., Howe, K., Hinerfeld, D., Ruan, X., et al. (2019). The Genome of C57BL/6J "Eve", the Mother of the Laboratory Mouse Genome Reference Strain. G3 9, 1795-1805.

41. Lippert, C., Casale, F.P., Rakitsch, B., and Stegle, O. (2014). LIMIX: genetic analysis of multiple traits. 003905 https://doi.org/10.1101/003905. 
42. McLaren, W., Gil, L., Hunt, S.E., Riat, H.S., Ritchie, G.R., Thormann, A., Flicek, P., and Cunningham, F. (2016). The Ensembl Variant Effect Predictor. Genome biology 17, 122.

43. Chang, Y.F., Imam, J.S., and Wilkinson, M.F. (2007). The nonsense-mediated decay RNA surveillance pathway. Annual review of biochemistry 76, 51-74.

44. Yang, H., Wang, J.R., Didion, J.P., Buus, R.J., Bell, T.A., Welsh, C.E., Bonhomme, F., Yu, A.H., Nachman, M.W., Pialek, J., et al. (2011). Subspecific origin and haplotype diversity in the laboratory mouse. Nature genetics 43, 648-655.

45. Wang, J.R., de Villena, F.P., and McMillan, L. (2012). Comparative analysis and visualization of multiple collinear genomes. BMC bioinformatics 13 Supp/ 3, S13.

46. Mulligan, M.K., Ponomarev, I., Boehm, S.L., 2nd, Owen, J.A., Levin, P.S., Berman, A.E., Blednov, Y.A., Crabbe, J.C., Williams, R.W., Miles, M.F., et al. (2008). Alcohol trait and transcriptional genomic analysis of C57BL/6 substrains. Genes, brain, and behavior 7, 677-689.

47. Kim, D., Paggi, J.M., Park, C., Bennett, C., and Salzberg, S.L. (2019). Graph-based genome alignment and genotyping with HISAT2 and HISAT-genotype. Nature biotechnology 37, 907-915.

48. Anders, S., Pyl, P.T., and Huber, W. (2015). HTSeq--a Python framework to work with high-throughput sequencing data. Bioinformatics 31, 166-169.

49. Chiang, C., Layer, R.M., Faust, G.G., Lindberg, M.R., Rose, D.B., Garrison, E.P., Marth, G.T., Quinlan, A.R., and Hall, I.M. (2015). SpeedSeq: ultra-fast personal genome analysis and interpretation. Nature methods 12, 966-968.

50. Faust, G.G., and Hall, I.M. (2014). SAMBLASTER: fast duplicate marking and structural variant read extraction. Bioinformatics 30, 2503-2505.

51. Tarasov, A., Vilella, A.J., Cuppen, E., Nijman, I.J., and Prins, P. (2015). Sambamba: fast processing of NGS alignment formats. Bioinformatics 31, 2032-2034.

52. Garrison, E., and Marth, G.T. (2012). Haplotype-based variant detection from short-read sequencing. arXiv:1207.3907 [q-bio.GN] https://arxiv.org/abs/1207.3907

53. Purcell, S., Neale, B., Todd-Brown, K., Thomas, L., Ferreira, M.A., Bender, D., Maller, J., Sklar, P., de Bakker, P.I., Daly, M.J., et al. (2007). PLINK: a tool set for whole-genome association and population-based linkage analyses. American journal of human genetics 81, 559-575.

54. Willems, T., Zielinski, D., Yuan, J., Gordon, A., Gymrek, M., and Erlich, Y. (2017). Genome-wide profiling of heritable and de novo STR variations. Nature methods 14, 590-592.

55. Benson, G. (1999). Tandem repeats finder: a program to analyze DNA sequences. Nucleic acids research 27, 573-580.

56. Layer, R.M., Chiang, C., Quinlan, A.R., and Hall, I.M. (2014). LUMPY: a probabilistic framework for structural variant discovery. Genome biology 15, R84.

57. Abyzov, A., Urban, A.E., Snyder, M., and Gerstein, M. (2011). CNVnator: an approach to discover, genotype, and characterize typical and atypical CNVs from family and population genome sequencing. Genome research 21, 974-984.

58. Pedersen, B.S., and Quinlan, A.R. (2018). Mosdepth: quick coverage calculation for genomes and exomes. Bioinformatics 34, 867-868. 